\title{
Models of void electromigration
}

\author{
L. J. CUMMINGS ${ }^{1}$, G. RICHARDSON ${ }^{1}$ and M. BEN AMAR ${ }^{2}$ \\ ${ }^{1}$ Laboratoire de Physique Statistique de l'ENS, \\ 24 Rue Lhomond, 75231 Paris Cedex 05, France $\dagger$ \\ email: Linda.Cummings@nottingham.ac.uk \\ ${ }^{2}$ Mechanical Engineering, 1-310, 77 Massachusetts Avenue, Cambridge MA 02139, USA
}

(Received 18 May 1999; revised 7 August 2000)

\begin{abstract}
We study the motion of voids in conductors subject to intense electrical current densities. We use a free-boundary model in which the flow of current around the insulating void is coupled to a law of motion (kinematic condition) for the void boundary. In the first part of the paper, we apply a new complex variable formulation of the model to an infinite domain and use this to (i) consider the stability of circular and flat front travelling waves, (ii) show that, in the unbounded metal domain, the only travelling waves of finite void area are circular, and (iii) consider possible static solutions. In the second part of the paper, we look at a conducting strip (which can be used to model interconnects in electronic devices) and use asymptotic methods to investigate the motion of long wavelength voids on the conductor boundary. In this case we derive a nonlinear parabolic PDE describing the evolution of the free boundary and, using this simpler model, are able to make some predictions about the evolution of the void over long times.
\end{abstract}

\section{Introduction}

Metal conductors subjected to intense electrical currents can exhibit the phenomenon of surface electromigration. A void can form within the conductor (nucleating either in the interior or at a boundary) and then 'drift' within the conductor, changing its shape as it does so. A schematic diagram of the process within a conducting metal strip is shown in Figure 1: Figure 1(a) depicts a void within a conductor, and Figure 1(b) a void at a conductor boundary. The conductors in question are thin in the dimension perpendicular to the paper and voids can penetrate fully in this direction in an almost two-dimensional fashion. Typical experimental configurations include the case in which the lower conductor boundary is sat on some rigid insulating substrate with the upper boundary open to the air (assumed insulating), and the case in which both boundaries are adjacent to rigid insulators. This drift of voids within conductors is due to the diffusion of atoms from one part of the void boundary to another, driven both by the electric field and by interfacial tension (which causes the atoms to move along the boundary to positions of large curvature).

The problem is of interest because such migration of voids within conductors is a relatively common form of circuit failure in electrical components. Experimental evidence

$\dagger$ Present address: School of Mathematics, University of Nottingham NG7 2RD, UK. 


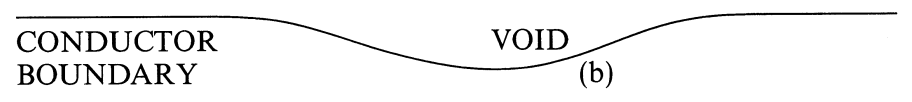

METAL FILM

(a)

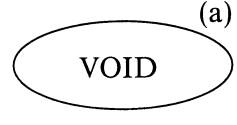

CONDUCTOR BOUNDARY

FIGURE 1. Schematic diagram of void electromigration within a conducting metal strip; (a) depicts a void within a conductor, and (b) a void at a conductor boundary.

suggests that for a long thin conductor subjected to a uniform electric field at its ends, a void at the conductor boundary will undergo fairly steady translation for a time, with a steepening profile, until some critical void shape is attained. At this point the void motion becomes visibly unstable; a narrow finger emerges from the void and propagates transversely to the electric field until it has traversed the conductor height, and circuit failure occurs [1]. Damage of this kind can be suppressed by covering the exposed surfaces of the conductor with a layer of glass ('passivating') but in this case approximately-circular voids nucleate in the interior of the conductor, and these can again deform and cause failure. Both geometries (voids at a conductor boundary, and voids in the interior of a conductor) are therefore of interest.

Most of the existing literature on this problem is either experimental [1], numerical simulations $[2,24,25,32]$, or concerned with circular or nearly-circular voids translating in infinite conducting media [17]. The only exact solutions which have been written down explicitly are (i) the uniformly translating circular void (fixed area) in the infinite domain, and (ii) a flat front parallel to the electric field [24], which models the situation at the edge of the conductor. Linear stability of (i) has been treated by several authors, e.g. [17, 32], and stability of (ii) by Schimschak \& Krug [24]. Numerical treatment of the 'narrow finger' instability mentioned above has been carried out recently by several authors [11, 27, 18, 19] (this problem has also been considered briefly in Suo et al. [30]). However, recent numerical evidence [2] points to the existence of other exact travellingwave solutions, in a strip geometry: finite-sized non-circular (though nearly so) voids, and an unbounded finger-shaped void (both symmetric about the strip centre-line) are found numerically, using a method similar to that used by McLean \& Saffman [16] to solve the Hele-Shaw fingering problem.

The layout of this paper is as follows. We begin by setting out the mathematical model in $\S 2$, and developing a new complex variable formulation in terms of the Schwarz function of the free (void) boundary and in terms of conformal mappings from some fixed domain onto the metal domain. We then consider simple exact solutions of the model in the infinite geometry: in this case it is shown that the only possible travelling-wave solution having finite void area is the circular travelling-wave. The linear stability analysis of these solutions is presented using our complex variable formulation, which is found to be the most efficient method. Our results, which are purely analytical, demonstrate that 
the circular void is unstable in the absence of surface tension effects, while a 'stagnation point'-type flat-front travelling wave can be either stable or unstable, depending on the direction of the current.

We also consider some possible solutions for static voids. In $\S 6$ we consider approximate solutions of the model, using methods of formal asymptotics. For a void at a conductor boundary (rather than in the interior of the metal), an approach similar to that used to describe surface waves on inviscid fluid of finite depth may be used. In the appropriate parameter régime we find that in the absence of surface tension effects, 'long-wavelength' voids are described by a first-order nonlinear wave equation, which can be solved semianalytically, and whose solutions are steepening waveforms. Inclusion of the surface tension terms adds a fourth-order (diffusion-type) term to this equation, which must then be solved numerically. This work extends that of Schimschak \& Krug [24], which considers only shallow voids at the conductor boundary. We present several solution types. Finally, we discuss the implications of our results and present our conclusions.

\section{Mathematical model}

We consider the standard two-dimensional model for the phenomenon, which is considered sufficient to describe most situations of interest. Conductors in real circuits tend to have dimensions length $\sim L$ (along the horizontal direction in Figure 1), depth $\sim D$ (into the paper in Figure 1), height $\sim h$ (in the vertical direction in figure 1) such that $L>>h>>D ; L$ is usually taken to be infinite, and so, sometimes, is $h$ (the void in the unbounded geometry, which describes small voids in the interior of a conductor). The two dimensions we consider are in the $(L, h)$-plane, quantities being averaged over the $D$-direction.

We denote the domain occupied by the metal by $\Omega(t)$, with boundary $\partial \Omega(t)$, oriented such that $\Omega$ lies to its left. In general, $\partial \Omega$ is composed of fixed insulating portions $\widehat{\partial \Omega}$, and the moving void boundary $\Gamma(t)$ (also assumed insulating, but with an additional kinematic boundary condition governing its motion). We shall usually suppress the time-dependence of the various functions, except where emphasis is needed. Our coordinate system $(x, y)$ is here taken such that the applied electric field is parallel to the $x$-direction, and where we consider the strip geometry, the strip is parallel to the $x$-axis. The mathematical model for the electric potential $\phi$ (where the electric field $\boldsymbol{E}=-\nabla \phi$ ) within the conductor is:

$$
\begin{array}{rr}
\nabla^{2} \phi=0 \quad \text { within } \Omega, \\
\frac{\partial \phi}{\partial n}=0 \quad \text { on } \partial \Omega=\widehat{\partial \Omega} \cup \Gamma, \\
-v_{n}=-Z^{*} e M \frac{\partial^{2} \phi}{\partial s^{2}}+M \omega \gamma \frac{\partial^{2} \kappa}{\partial s^{2}} \quad \text { on } \Gamma, \\
\phi_{x} \sim E_{\infty} \quad \text { as } x \rightarrow+\infty, \\
\phi_{x} \sim \text { const. } \quad \text { as } x \rightarrow-\infty, \\
\Gamma(0) \quad \text { specified, }
\end{array}
$$

where $\partial / \partial n$ denotes the derivative in the direction of the outward normal $\boldsymbol{n}$ to $\partial \Omega$ (pointing away from $\Omega$ ) and $\partial / \partial s$ denotes the derivative with respect to arclength $s$ along $\Gamma$. The 


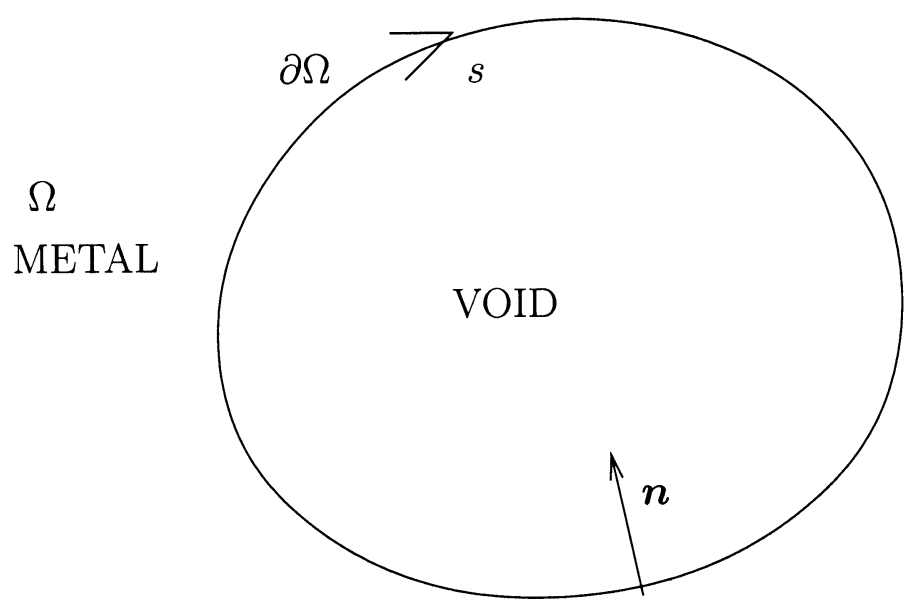

FIGURE 2. The conventions adopted in the model (2.1)-(2.3).

conventions adopted are indicated in Figure 2. In the above, $v_{n}$ is the velocity of $\Gamma$ in the direction of $\boldsymbol{n}, Z^{*} e$ is the effective charge of a metal ion, $M$ is the adatom mobility, $\omega$ is the atomic volume, $\gamma$ is the coefficient of surface tension, and $\kappa$ is the curvature of $\Gamma$, taken to be positive for a void of convex shape ( e.g. a circle). All of the physical parameters are assumed to be constant in our model.

The far-field behaviour specified in (2.4) assumes a uniform electric field $\boldsymbol{E}=-E_{\infty} \boldsymbol{i}$ applied at infinity, the case we shall usually consider in this paper (though different behaviour can also be envisaged; for example in the local problems near the tip of a void, or describing the field at the junction of two or more conductors (see \$4.2)). The value of the constant in (2.5) depends upon the geometry assumed at minus infinity; it must be consistent with current conservation. The effective charge $Z^{*} e$ of a metal ion can be either positive or negative, due to the fact that there are two competing forces acting on the ions: the direct electrostatic interaction, which pushes the positively-charged ions towards the cathode, and the so-called 'electron wind' force, due to collisions of the electrons (which are moving towards the anode) with the ions. Each of these effects produces a force on the ions proportional to the applied electric field, hence the two effects can be combined as a single effective force on the ion, equal to the electric field strength multiplied by the 'effective charge' of the ion. In most common conductors (e.g. aluminium) the electron wind force dominates, and the value of $Z^{*} e$ is negative; we shall assume this in our model.

Physically the first term on the right-hand side of (2.3) represents surface diffusion of atoms under the influence of the electric field, in the direction of the electron flow when $Z^{*} e<0$. In this case, the void moves in the direction opposite to the electron flow, that is, in the sense of the electric field. The second term on the right-hand side of (2.3) is less obvious, but acts to minimise the surface energy $G$,

$$
G=\int_{\Gamma} \gamma d s=\gamma \times \operatorname{length}(\Gamma),
$$

subject to the constraint that the area enclosed by $\Gamma$, if finite, is conserved. (A derivation of this law of motion by surface diffusion, which was first introduced by Mullins [21], is given in Davi \& Gurtin [5]; see Elliott \& Garcke [7] and the references therein for 
a discussion of general laws of diffusive surface motion). For voids of finite area, the constant area constraint is equivalent to the condition that there is to be no net creation or destruction of metal; without this constraint one would just get the kinematic boundary condition $-v_{n}=-Z^{*} e M \phi_{s s}-\gamma \kappa$. If $\Gamma$ extends to infinity, and the void area is infinite, the condition still ensures conservation of metal mass, which is clearly always desirable on physical grounds. Note that without loss of generality we may take $Z^{*} e<0$, since if it is positive we can just replace $\phi$ by $-\phi$, and work with $-Z^{*} e<0$. We may also assume $E_{\infty}>0$ by a similar argument, for if not we can replace $x$ by $-x$ and solve the problem for $-E_{\infty}$. In any case, the signs of these parameters only influence which way the voids move within the conductor; with our conventions they move from right to left (this can be seen most clearly from the direct analysis of $\S 6$ ).

Before proceeding further, we nondimensionalise the problem, following Ben Amar [2]. We have four relevant dimensional physical parameters: $Z^{*} e, M, \gamma$ and $E_{\infty}$ whose dimensions are given in Appendix A; suppose also that $l$ and $\tau$ are typical length- and time-scales (to be determined). If surface tension effects are dominant in the problem, $l$ must be given by $l=E_{\infty}\left|Z^{*} e\right| / \gamma$, since this combination of parameters is the only one having the dimensions of length. Otherwise, $l$ is determined by the geometry; for instance, if the void size is comparable to the conductor width $2 h$, we take $l=h$. In either case, the characteristic timescale is then given by $\tau=l^{2} /\left(E_{\infty}\left|Z^{*} e\right| M\right)$. Thus we scale $x$ and $y$ with $l$, time with $\tau$, $\phi$ with $E_{\infty} l,{ }^{1}$ which results in the dimensionless model:

$$
\begin{gathered}
\nabla^{2} \phi=0 \quad \text { within } \Omega, \\
\frac{\partial \phi}{\partial n}=0 \quad \text { on } \partial \Omega=\partial \hat{\Omega} \cup \Gamma, \\
-v_{n}=\frac{\partial^{2} \phi}{\partial s^{2}}+\sigma \frac{\partial^{2} \kappa}{\partial s^{2}} \quad \text { on } \Gamma, \\
\phi \sim x \quad \text { as } x \rightarrow+\infty,
\end{gathered}
$$

where the dimensionless parameter $\sigma$ is given by

$$
\sigma=\frac{\omega \gamma}{E_{\infty}\left|Z^{*} e\right| l^{2}}
$$

and measures the relative effects of surface diffusion due to the surface tension, and that due to the electric field. For problems with $\sigma \ll 1$ we may expect the void motion to be approximated by the (much simpler) Zero Surface Tension (ZST) model, with $\sigma=0$. (Note that the ZST problem is time-reversible if we also change the sign of $\phi$. Thus, given any ZST solution, there is an equivalent solution with the electric field and the free boundary motion both reversed.)

\section{Complex variable formulation}

Since we have a two-dimensional Laplacian moving boundary problem we may consider a complex variable approach; such methods have proved invaluable in studying similar problems such as classical Hele-Shaw flow [14]. Thus we set $z=x+i y$ and introduce a complex electrical potential $w(z)=\phi(x, y)+i \psi(x, y)$, where $\psi$ is a harmonic conjugate of

1 A different scaling for $\phi$ may be appropriate if different behaviour at infinity is specified. 
$\phi .^{2}$ We note here that a complex variable approach, considering $w$ as an analytic function of $z$, has recently been considered by Bradley et al. [3] for an electromigration problem in a strip. However, the situation there is different to that we consider, in that the current is applied across the width of the strip rather than along its length (the sides $y= \pm 1$ of the conducting strip are taken to be equipotentials). They consider a slit-shaped void along the length of the strip. In this case, the problem reduces to a simple Hele-Shaw crack problem which can be solved explicitly - the electric potential $\phi$ in their solution is easily checked to be equivalent to the streamfunction for the zero-width $(\lambda=0)$ Saffman-Taylor finger solution [23]. This same approach was used earlier by Sornette \& Vanneste [28, 29], on an equivalent problem in rupture in thermal fuse networks. Our aim is to formulate a more general complex variable approach.

An important concept we shall need is that of the Schwarz function of an analytic curve $\Gamma(t)$ : this is the unique complex function $g(z, t)$, analytic in some neighbourhood of $\Gamma(t)$, such that the equation $\bar{z}=g(z, t)$ describes the curve. We remark at the outset that there are severe restrictions on a general complex function $g$ if it is to be a Schwarz function. On the curve we require

$$
z=\overline{g(z)}=\bar{g}(\bar{z})=\bar{g}(g(z))=(\bar{g} \circ g)(z),
$$

where $\bar{g}$ is the complex conjugate function of $g$, defined by $\bar{g}(z)=\overline{g(\bar{z})}$. Both sides of this equality are functions analytic in some neighbourhood of the curve, so the equality may be deduced to hold everywhere, by complex analytic continuation. Hence, if $g$ is to be the Schwarz function of an analytic curve, we require $\bar{g}$ to be its inverse.

It can be shown [6] that geometrical properties of the curve may be simply expressed in terms of $g$; with the direction conventions established in Figure 2, and with prime denoting $\partial / \partial z$ we have

$$
v_{n}=\frac{-i}{2 \sqrt{g^{\prime}(z, t)}} \frac{\partial g}{\partial t}(z, t), \quad \kappa=i \frac{\partial}{\partial z}\left(\frac{1}{\sqrt{g^{\prime}(z, t)}}\right), \quad \frac{\partial z}{\partial s}=\frac{1}{\sqrt{g^{\prime}(z, t)}},
$$

thus on $\Gamma, \partial / \partial s=(\partial / \partial z) / \sqrt{g^{\prime}(z, t)}$. (The above expressions are readily verified for the simple example of a circle of radius $\sqrt{t}$, for which $g(z, t)=t / z$.) On the void boundary $\Gamma(t),(2.8)$ implies that

$$
\frac{\partial^{2} \phi}{\partial s^{2}}=\frac{\partial^{2} w}{\partial s^{2}}
$$

Putting all this together, we can express the boundary condition (2.9) holding on $\Gamma$ in complex variable form as:

$$
\frac{i}{2} \frac{\partial g}{\partial t}=\frac{\partial}{\partial z}\left(\frac{1}{\sqrt{g^{\prime}(z)}} \frac{\partial}{\partial z}\left[w(z)+i \sigma \frac{\partial}{\partial z}\left(\frac{1}{\sqrt{g^{\prime}(z)}}\right)\right]\right) .
$$

Since $w$ is analytic throughout $\Omega$ (except at infinity), and $g$ is known to be analytic at least in some neighbourhood of the void boundary $\Gamma$ (assumed to be an analytic curve), this relation may be analytically continued [4] away from $\Gamma$, and deduced to hold wherever the various quantities are defined.

2 The function $\psi(x, y)$ has no real physical significance, but does have the property that its level curves are parallel to the instantaneous electric field direction at any instant of time, $c f$ the streamfunction in fluid dynamics. 
If the asymptotic behaviour is as in (2.10), $w$ has the behaviour

$$
w(z) \sim z \quad \text { as } \mathfrak{R}(z) \rightarrow+\infty .
$$

Thus, without the added complication of fixed insulating boundaries to be dealt with (i.e. in the unbounded geometry) one may seek solutions for a given shape of void by: (i) finding the Schwarz function $g$ of the postulated void shape (e.g. circle, ellipse, parabola,...), and (ii) attempting to match the behaviour at infinity and eliminate any other singular behaviour of $w$ within $\Omega$ in equation (3.1). If one wishes to consider solutions in a strip geometry $-1 \leqslant y \leqslant 1$ using this approach, one has to use a periodic array of voids, so that the insulating conditions on $y= \pm 1$ are satisfied automatically.

\subsection{Conformal mapping}

Another approach which can be helpful is to introduce a time-dependent conformal map $z=f(\zeta, t)$ from some canonical domain in an auxiliary complex $\zeta$-plane, onto $\Omega$. Often in the following we shall drop the explicit time-dependence, writing $f(\zeta)$. The two simplest cases to envisage are (i) a finite void translating in an unbounded metal domain, and (ii) a semi-infinite void moving through a semi-infinite metal domain, though periodic generalisations of each of these cases (corresponding to a finite or infinite void (e.g. finger) in a conducting strip) are also feasible. In case (i) the most appropriate canonical domain to map from is the unit disc, with $\zeta=0$ mapping to infinity such that

$$
f(\zeta) \sim \frac{a}{\zeta} \quad \text { as } \zeta \rightarrow 0
$$

in case (ii) it is the right-half plane, with asymptotic behaviour

$$
F(\zeta) \sim A \zeta \quad \text { as } \zeta \rightarrow \infty
$$

for a void boundary which is asymptotically flat, or

$$
F(\zeta) \sim A \zeta^{2} \text { as } \zeta \rightarrow \infty
$$

for a void boundary which is asymptotically parabolic, etc. We now consider each case separately.

\subsubsection{Mapping from the unit disc}

Consider a time-dependent conformal map from the unit disc in an auxiliary complex $\zeta$-plane onto the metal domain $\Omega(t) \subset \mathbb{C}, z=f(\zeta, t)$. This is always possible by the Riemann mapping theorem; moreover if one insists that the origin $\zeta=0$ map to infinity in the $z$-plane, and that the negative real axis near $\zeta=0$ correspond to the positive real axis near $z=\infty$, then the mapping is unique [4]. The free (void) boundary is then the image under $f$ of $|\zeta|=1$, so on the boundary we have

$$
g(z)=\bar{z}=\overline{f(\zeta)}=\bar{f}(\bar{\zeta})=\bar{f}(1 / \zeta) ;
$$

since the functions on the extreme left- and right-hand sides of this equality are analytic in some neighbourhood of the boundary, the equality

$$
g(z)=\bar{f}(1 / \zeta)
$$


holds globally by analytic continuation. Using this and the relations

$$
\frac{\partial}{\partial z}=\frac{1}{f^{\prime}(\zeta)} \frac{\partial}{\partial \zeta}, \quad \frac{\partial \zeta}{\partial t}=-\frac{f_{t}(\zeta)}{f^{\prime}(\zeta)},
$$

where the prime denotes $\partial / \partial \zeta$ and the subscript $t$ denotes $\partial / \partial t$, we can thus reformulate equation (3.1) as a functional differential equation in the $\zeta$-plane:

$$
\begin{aligned}
& \frac{1}{\zeta^{2}} f_{t}(\zeta) \bar{f}^{\prime}(1 / \zeta)+\bar{f}_{t}(1 / \zeta) f^{\prime}(\zeta)=-2 \frac{\partial}{\partial \zeta}\left[\frac{\zeta W^{\prime}(\zeta)}{\left(f^{\prime}(\zeta) \bar{f}^{\prime}(1 / \zeta)\right)^{1 / 2}}\right] \\
& \quad-2 \sigma \frac{\partial}{\partial \zeta}\left\{\frac{\zeta}{\left(f^{\prime}(\zeta) \bar{f}^{\prime}(1 / \zeta)\right)^{1 / 2}} \frac{\partial}{\partial \zeta}\left[\frac{1}{f^{\prime}(\zeta)} \frac{\partial}{\partial \zeta}\left(\zeta\left(\frac{f^{\prime}(\zeta)}{\bar{f}^{\prime}(1 / \zeta)}\right)^{1 / 2}\right)\right]\right\} .
\end{aligned}
$$

Care is needed when choosing the branches of the square-roots on the right-hand side of (3.4). Here, $W(\zeta) \equiv w(f(\zeta))$ is the complex potential in the $\zeta$-plane, which may be written down explicitly for a given problem. For example, if we have a finite void translating within an unbounded domain, then for $f$ to map conformally onto $\Omega$ for $|z| \gg 1$ we must have the behaviour $f(\zeta) \sim a / \zeta$ as $\zeta \rightarrow 0$, for some constant $a>0$. From (3.2) and (2.8) $W$ thus satisfies

$$
W(\zeta) \sim \frac{a}{\zeta} \quad \text { as } \zeta \rightarrow 0, \quad \Im(W)=0 \quad \text { on }|\zeta|=1,
$$

and hence

$$
W(\zeta)=a\left(\zeta+\frac{1}{\zeta}\right)
$$

\subsubsection{Mapping from a half-plane}

Here we write the map as $z=F(\zeta, t)$ to distinguish from the unit disc case. If we write $\zeta=\xi+i \varsigma$ then the void boundary is given by $z=F(i \varsigma), \varsigma \in \mathbb{R}$, so on the boundary we have:

$$
g(z)=\bar{z}=\overline{F(\zeta)}=\bar{F}(\bar{\zeta})=\bar{F}(-\zeta) .
$$

Thus reasoning as for the unit disc we deduce that

$$
g(z)=\bar{F}(-\zeta),
$$

and we can again reformulate equation (3.1) as a functional differential equation holding in the $\zeta$-plane:

$$
\begin{aligned}
& F_{t}(\zeta) \bar{F}^{\prime}(-\zeta)+\bar{F}_{t}(-\zeta) F^{\prime}(\zeta)=-2 \frac{\partial}{\partial \zeta}\left[\frac{W^{\prime}(\zeta)}{\left(F^{\prime}(\zeta) \bar{F}^{\prime}(-\zeta)\right)^{1 / 2}}\right] \\
& \quad-2 \sigma \frac{\partial}{\partial \zeta}\left\{\frac{1}{\left(F^{\prime}(\zeta) \bar{F}^{\prime}(-\zeta)\right)^{1 / 2}} \frac{\partial}{\partial \zeta}\left[\frac{1}{F^{\prime}(\zeta)} \frac{\partial}{\partial \zeta}\left(\left(\frac{F^{\prime}(\zeta)}{\bar{F}^{\prime}(-\zeta)}\right)^{1 / 2}\right)\right]\right\} .
\end{aligned}
$$

Again, because of the fixed domain in $\zeta$-space, $W(\zeta)$ may be written down explicitly for a given problem, though it will depend upon the asymptotic behaviour of both $w$ and $F$.

Suppose we seek a solution with an asymptotically-flat boundary; then we must have $F(\zeta) \sim A \zeta$ as $\zeta \rightarrow \infty$, for some constant $A$. For behaviour at infinity given by (3.2) (and 
with (2.8) also to be satisfied) we then require

$$
W(\zeta) \sim A \zeta \quad \text { as } \zeta \rightarrow \infty, \quad \mathfrak{I}(W)=0 \quad \text { on } \mathfrak{R}(\zeta)=0,
$$

and hence

$$
W(\zeta)=A \zeta
$$

where $A=i B$ for some $B \in \mathbb{R}$. (This indicates that the asymptotically-flat boundary lies parallel to the electric field, since the far-field behaviour of the map $F$ is $F(\zeta) \sim i B \zeta$ and we are mapping from the right-half $\zeta$-plane; this is the case considered by Schimschak $\&$ Krug [24]). However, if we are interested in a flat-front type solution as describing the situation near the 'nose' of a translating void, then the appropriate far-field behaviour for $w$ is

$$
w(z) \sim z^{2} \quad \text { as }|z| \rightarrow \infty,
$$

( $c f$ a stagnation-point flow in fluid dynamics). The solution for $W$ is then given by

$$
W(\zeta)=A^{2} \zeta^{2},
$$

where now $A^{2}$ is required to be real if the boundary condition (2.8) is to hold.

For a solution where the void boundary is asymptotically parabolic, $F(\zeta) \sim A^{2} \zeta^{2}$ as $\zeta \rightarrow \infty$ for some $A$. In this case, with $w$ satisfying (3.2) (instead of (3.8)), the solution for $W$ is again given by (3.9), since this satisfies

$$
w(z)=W(\zeta)=A^{2} \zeta^{2} \sim F(\zeta)=z, \quad \text { for }|z| \gg 1,|\zeta| \gg 1 .
$$

\section{Exact solutions}

\subsection{Travelling-wave solutions}

We now consider some simple travelling-wave solutions to the model using the complex variable approach. Consider first the travelling-wave version of (3.1). The Schwarz function $g(z, t)$ must be of the form

$$
g(z, t)=-\bar{V} t+g_{0}(z+V t),
$$

where $g_{0}$ is the Schwarz function for the shape of the free boundary in the travelling-wave frame. In this frame, with $\hat{z}=z+V t$ (where possibly $V \in \mathbb{C}$ ) and functional dependence only on $\hat{z},(3.1)$ becomes:

$$
\sqrt{g_{0}^{\prime}(\hat{z})}\left(\bar{V} \hat{z}-V g_{0}(\hat{z})\right)+2 \sigma \frac{d^{2}}{d \hat{z}^{2}}\left(\frac{1}{\sqrt{g_{0}^{\prime}(\hat{z})}}\right)=2 i \frac{d w}{d \hat{z}} ;
$$

the velocity of the travelling wave is $(-\mathfrak{R}(V),-\mathfrak{I}(V))$. Even in the ZST case, with $\sigma=0$, solutions to this model are difficult to find, because $w$ is required to be analytic throughout $\Omega$, yet the square-root of $g_{0}^{\prime}$ on the left-hand side means that most hypothetical free boundary shapes will introduce square-root branch-points into $w$ and therefore not give acceptable solutions. However, two simple solutions may be written down immediately. 


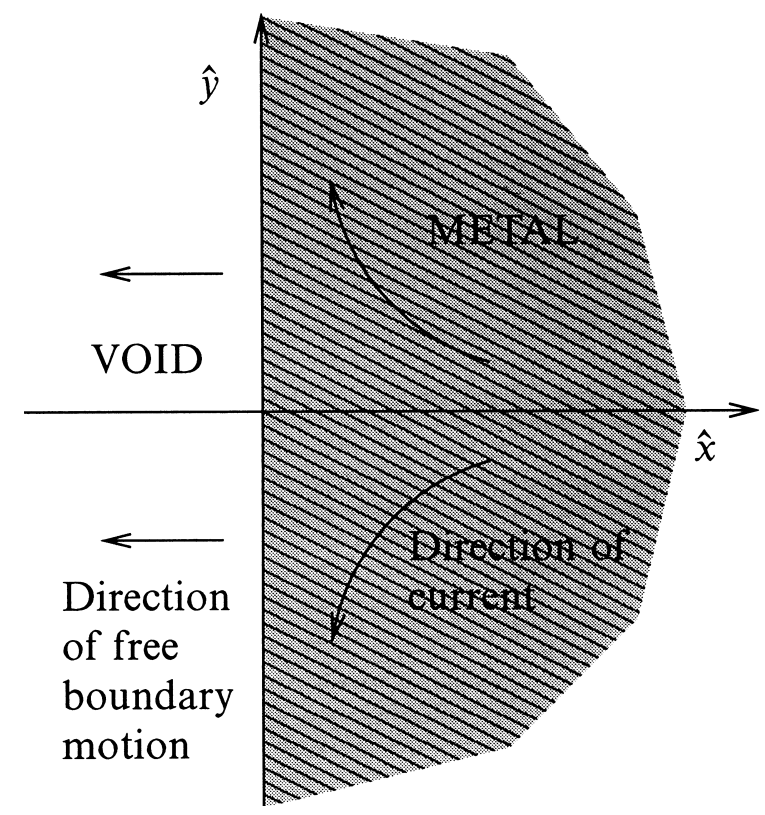

FiguRE 3. The geometry of the flat-front travelling-wave. The metal occupies the shaded region, and the arrows there indicate the direction of the electric field. The arrows in the void region indicate the direction of the free boundary motion.

\subsubsection{Circular travelling-wave void}

The Schwarz function for a circular void of radius a centred on the origin is just $g_{0}(\hat{z})=a^{2} / \hat{z}$, which in (4.1), with $V$ assumed real (this is easily seen to be necessary to satisfy the asymptotic behaviour (3.2)), leads to

$$
w(\hat{z})=\frac{a V}{2}\left(\hat{z}+\frac{a^{2}}{\hat{z}}\right) .
$$

Thus if we are to have $w(z) \sim z$ at infinity the void must move from right to left with a velocity of $2 / a$. This solution was first presented by Ho [13].

\subsubsection{Flat-front travelling-wave}

The Schwarz function for the straight line $m y=x$ is just $g(z)=z(m-i) /(m+i)$. Thus (4.1) gives the complex electric potential necessary for a solution as

$$
w(\hat{z})=\frac{\hat{z}^{2}}{4} \frac{(m-i)^{1 / 2}}{\left(m^{2}+1\right)^{3 / 2}}((\bar{V}+V)-i m(\bar{V}-V)) .
$$

This corresponds to the situation of asymptotic behaviour (3.8), hence for $w$ to be a real multiple of $\hat{z}^{2}$ we require $m=0$. Thus in fact $w(\hat{z})= \pm \hat{z}^{2}$ and $V= \pm 2$. The geometry of the solution with the ' + ' signs is sketched in Figure 3; for the solution with the '-' signs the directions on all the arrows are reversed. These solutions model the local behaviour at the right-hand and left-hand edges (the rear and front, relative to the direction of travel) of a closed void (such as the circular solution found above) within a conductor. 
It is easily checked from (4.1) that there is no travelling wave parabola, for which $g_{0}(\hat{z})=\hat{z}-4 a+4 a \sqrt{ }(1-\hat{z} / a)$; this would lead to an unphysical square-root singularity in $w$.

\subsubsection{Stability analyses for $\sigma=0$}

In this section we consider the linear stability of the travelling wave solutions found above. We do this in the absence of surface tension effects, although both solutions are valid for arbitrary $\sigma$. The most appropriate forms of the governing equations for this purpose are (3.4) and (3.6) (with $\sigma=0$ ). We do the circular void first, which, as mentioned in the Introduction, has been considered by Mahadevan \& Bradley [17] and Wang et al. [32].

Setting $a=1$ and $V=2$ for convenience in (4.2), the basic solution is given by

$$
f(\zeta, t)=-2 t+\frac{1}{\zeta}, \quad W(\zeta)=\zeta+\frac{1}{\zeta} .
$$

We seek a perturbation of the form

$$
f(\zeta, t)=-2 t+\frac{1}{\zeta}+\epsilon v(\zeta, t),
$$

for some function $v(\zeta, t)$ analytic on the unit disc, which must be expressible as a convergent sum of the form

$$
v(\zeta, t)=\sum_{k=0}^{\infty} d_{k}(t) \zeta^{k},
$$

on the unit disc (there can be no $k=-1$ term if the void is to conserve its area). Since the void boundary is the image of $\zeta=e^{i \theta}(\theta \in(0,2 \pi))$, and the $d_{k}(t)$ are complex, it is easy to see how the representation of (4.5) corresponds to a harmonic perturbation to the free boundary as considered in a conventional stability analysis: taking real and imaginary parts in $x(\theta, t)+i y(\theta, t)=f\left(e^{i \theta}, t\right)$ gives

$$
\begin{array}{r}
x(\theta, t)=-2 t+\cos \theta+\epsilon \sum_{k=0}^{\infty}\left(\mathfrak{R}\left(d_{k}(t)\right) \cos (k \theta)-\mathfrak{J}\left(d_{k}(t)\right) \sin (k \theta)\right), \\
y(\theta, t)=-\sin \theta+\epsilon \sum_{k=0}^{\infty}\left(\mathfrak{R}\left(d_{k}(t)\right) \sin (k \theta)+\mathfrak{J}\left(d_{k}(t)\right) \cos (k \theta)\right) .
\end{array}
$$

The complex potential in the $\zeta$-plane, $W(\zeta)$, must remain unchanged in order to satisfy the condition that its imaginary part vanish on $|\zeta|=1$ (but the potential in the $z$-plane, $w(z)$, must change as the solution is perturbed); this is why tackling the problem in the $\zeta$-plane is so appropriate. Substituting for $W$ and the perturbed $f$ in (3.4) the leading orders match automatically (on choosing the appropriate sign for the square-root on the right-hand side of (3.4)), and equating the terms of order $\epsilon$ on both sides yields two equations for $v$ :

$$
\frac{\partial}{\partial \zeta}\left(\zeta\left(\zeta^{2}-1\right) \frac{\partial v}{\partial \zeta}\right)+2 \frac{\partial v}{\partial \zeta}+\frac{\partial v}{\partial t}=0,
$$

and an equivalent independent equation for $\bar{v}(1 / \zeta)$. A conventional stability analysis would involve assuming a particular time-dependence $\exp (\mu t)$ for the perturbation in (4.6) 
and solving the resulting eigenvalue problem for $\mu$. This is difficult here due to the form of the differential operator in (4.6), hence we adopt the following non-standard approach. Direct substitution of (4.5) in (4.6) yields

$$
\begin{aligned}
& O(1): \quad d_{1}+\dot{d}_{0}=0, \\
& O(\zeta): \quad \dot{d}_{1}=0 \text {, } \\
& O\left(\zeta^{k}\right): \quad\left(k^{2}-1\right)\left(d_{k-1}-d_{k+1}\right)+\dot{d}_{k}=0, \quad k \geqslant 2 .
\end{aligned}
$$

The first two equations can clearly be solved immediately, giving

$$
d_{1}(t)=d_{1}(0), \quad d_{0}(t)=d_{0}(0)-d_{1}(0) t,
$$

and we may decouple these modes from the remainder of the terms by defining

$$
D_{2 m+1}(t):=d_{2 m+1}(t)-d_{1}(0), \quad D_{2 m}(t):=d_{2 m}(t),
$$

for $m \geqslant 1$. Then the function

$$
N(\zeta, t)=\sum_{k=2}^{\infty} D_{k}(t) \zeta^{k} \equiv \sum_{k=2}^{\infty} d_{k}(t) \zeta^{k}-\frac{d_{1}(0) \zeta^{3}}{1-\zeta^{2}},
$$

satisfies the same equation (4.6), for $|\zeta|<1$. Note that $|\zeta|=1$ is the circle of convergence for $N(\zeta, t)$; as $\zeta \rightarrow 1$ we have the local behaviour

$$
N(\zeta, t) \sim-\frac{d_{1}(0)}{2(1-\zeta)}+O(1)
$$

since the sum $\sum_{k=2}^{\infty} d_{k}(t) \zeta^{k}$ is assumed convergent on the unit disc. We may write equation (4.6) for $N$ in self-adjoint form as

$$
\frac{\partial}{\partial \zeta}\left[\frac{\left(\zeta^{2}-1\right)^{2}}{\zeta} \frac{\partial N}{\partial \zeta}\right]+\frac{\left(\zeta^{2}-1\right)}{\zeta^{2}} \frac{\partial N}{\partial t}=0,
$$

valid for $|\zeta|<1$ (the singularity at $\zeta=0$ is removeable with the form for $N(\zeta, t)$ in $(4.7)$ ). We now restrict attention to the interval $(0,1)$ of the real axis (where (4.9) must certainly hold, since it holds on the entire unit disc) and make the substitution $\zeta=e^{-x}, x \in(0, \infty)$. Writing $\mathscr{N}(x, t)=N(\zeta, t)$ on this interval gives the equation for $\mathscr{N}$ as

$$
2 \frac{\partial}{\partial x}\left(\frac{\partial \mathscr{N}}{\partial x} \sinh ^{2} x\right)=\frac{\partial \mathscr{N}}{\partial t} \sinh x
$$

and finally, writing $B(x, t)=\mathscr{N}(x, t) \sinh x$ gives

$$
\frac{\partial B}{\partial t}=2 \sinh x\left(\frac{\partial^{2} B}{\partial x^{2}}-B\right) .
$$

The boundary conditions on $B(x)$ are imposed at $x=0(\zeta=1)$ and $x=\infty(\zeta=0)$. Since by (4.8) $\mathscr{N}(x, t) \sim-d_{1}(0) /(2 x)$ as $x \rightarrow 0$, we have $B(0, t)=-d_{1}(0) / 2$, and since by (4.7) $N(\zeta, t) \sim D_{2}(t) \zeta^{2}$ as $\zeta \rightarrow 0, \mathscr{N}(x, t) \sim D_{2}(t) e^{-2 x}$ as $x \rightarrow \infty$, and so $B(x, t) \rightarrow 0$ as $x \rightarrow \infty$. Thus we have to solve the diffusion equation (4.10) subject to these boundary conditions:

$$
B(0, t)=-\frac{d_{1}(0)}{2}, \quad B(\infty, t)=0,
$$


and an initial condition, which is readily verified to be

$$
B(x, 0)=\sum_{k=2}^{\infty} d_{k}(0) e^{-k x} \sinh x-\frac{d_{1}(0)}{2} e^{-2 x} .
$$

As remarked, (4.10) is a diffusion equation on $(0, \infty)$, with positive diffusion coefficient $\sinh x$. Thus as $t \rightarrow \infty$ we expect that $B(x, t)$ tends to the steady-state solution satisfying both boundary conditions, namely

$$
B(x, \infty)=-\frac{d_{1}(0)}{2} e^{-x} .
$$

Defining $\hat{B}(x, t)=B(x, t)+d_{1}(0) e^{-x} / 2, \hat{B}$ satisfies (4.10) plus homogeneous boundary conditions $\hat{B}(0, t)=0=\hat{B}(\infty, t)$. Multiplying equation (4.10) for $\hat{B}$ through by $\hat{B}$ and integrating between $x=0$ and $x=\infty$, using these boundary conditions, we find

$$
\frac{1}{2} \frac{d}{d t} \int_{0}^{\infty} \frac{\hat{B}^{2}}{\sinh x} d x=-2 \int_{0}^{\infty}\left(\hat{B}_{x}^{2}+\hat{B}^{2}\right) d x<0 .
$$

This reveals $\hat{B}$ to be decreasing in time, implying that $B$ does approach the steady-state solution as $t \rightarrow \infty$. Thus

$$
\begin{aligned}
\mathscr{N}(x, t) & \rightarrow-\frac{d_{1}(0) e^{-x}}{e^{x}-e^{-x}}, \\
\Rightarrow N(\zeta, t) & \rightarrow-\frac{d_{1}(0) \zeta^{2}}{1-\zeta^{2}}=-d_{1}(0) \sum_{k=1}^{\infty} \zeta^{2 k} .
\end{aligned}
$$

Here, (4.11) holds not only on the real interval $0<\zeta<1$, but on the entire open unit disc $|\zeta|<1$. This is because the function $N(\zeta, t)$ is known to be complex analytic on $|\zeta|<1$, thus if it is known on a dense subset of the unit disc (the interval $0<\mathfrak{R}(\zeta)<1$ ), this result can be analytically continued [4] to the whole unit disc. It follows that

$$
D_{2 k} \rightarrow-d_{1}(0), \quad D_{2 k+1} \rightarrow 0 \quad(k \geqslant 1),
$$

as $t \rightarrow \infty$, and

$$
d_{k}(t) \rightarrow(-1)^{k+1} d_{1}(0) \quad k \geqslant 2,
$$

as $t \rightarrow \infty$. Thus the sum (4.5) representing the perturbation to the circular boundary slowly becomes divergent as $t$ increases, since

$$
\begin{aligned}
v(\zeta, t) & \sim\left(d_{0}(0)-d_{1}(0) t\right)-d_{1}(0) \sum_{k=1}^{\infty}(-1)^{k} \zeta^{k}, \quad t \gg 1 \\
& =\left(d_{0}(0)-d_{1}(0) t\right)+\frac{d_{1}(0) \zeta}{1+\zeta},
\end{aligned}
$$

which is unbounded at $\zeta=-1$. So, the perturbed circular void is unstable in the absence of surface tension effects. The linear analysis obviously breaks down before the above divergent sum, which for $|\zeta|=1$ represents a circular void with a 'spike' at its front (with respect to the direction of motion, so the left-hand side), is closely approached. However we may conjecture that in the absence of surface tension the initial destabilisation of the void occurs via a protruberance (or dimple, in the case $\mathfrak{R}\left(d_{1}(0)\right)<0$ ) forming at this 
forward-most point (the image of $\zeta=-1$ ). The fact that the coefficient $d_{1}(0)$ is arbitrary and complex corresponds to the fact that the protruberance/dimple may start to grow in any direction from this point. In the 'spike' limit, the angle made by the spike with the axis of translation is $\left.\tan ^{-1}\left(\mathfrak{I}\left(d_{1}(0)\right) / \mathfrak{R}\left(d_{1}(0)\right)\right)\right)$, hence we expect this to be the initial direction of growth. Both scenarios have been observed in numerical simulations on circular voids; protruberances in Mahadevan \& Bradley [17] and Schimschak \& Krug [25], and dimples in Li et al. [15] and Schimschak \& Krug [25]. Both cases are of interest: in the former case, the growth direction is arbitrary, hence the instability may mark the onset of a finger-shaped void propagating at an angle to the electric field such as those observed in experiments [1] and simulations [18, 19] of conductor failure. In the latter case, a dimple pointing into the void may herald the onset of void break-up via so-called 'invagination', which has been observed in the simulations of Li et al. [15] and Schimschak \& Krug [25].

Now consider the simple flat-front solutions, for which the stability analysis is more straightforward. The solution of Figure 3 is given in the notation of (3.6) by $F(\zeta, t)=$ $-2 t+\zeta, W(\zeta)=\zeta^{2}$; again we have normalised so that we have a wave of speed 2 . We perturb this solution, writing

$$
F(\zeta, t)=-2 t+\zeta+\epsilon v(\zeta, t) .
$$

Substituting in (3.6), the leading-order terms match automatically, and equating the $O(\epsilon)$ terms gives

$$
\frac{\partial}{\partial \zeta}\left(\zeta^{2} \frac{\partial v}{\partial \zeta}\right)-\frac{\zeta}{2} \frac{\partial v}{\partial t}=0
$$

(plus an equivalent equation for $\bar{v}(-\zeta, t)$ ) after recasting in self-adjoint form. This equation holds for all $\mathfrak{R}(\zeta)>0$. We now restrict attention to the positive $\xi$-axis $(\zeta=\xi+i \varsigma)$, writing $\xi v(\xi)=B(\xi)$ there; $B$ then satisfies the diffusion-type equation

$$
\frac{\partial B}{\partial t}=2 \xi \frac{\partial^{2} B}{\partial \xi^{2}}, \quad \xi \geqslant 0 .
$$

For the stability analysis we seek a perturbation $v$ such that on the boundary

$$
v(i \varsigma, t)=\sum_{k=1}^{\infty} d_{k}(t) e^{-i k \varsigma}=\sum_{k=1}^{\infty}\left(d_{k}(t) e^{-k \zeta}\right)_{\xi=0}
$$

(we need the minus sign in the exponent for $v$ to represent an acceptable perturbation to $F$ as $\mathfrak{R}(\zeta) \rightarrow \infty$; as with the circular void, this can be seen to represent a harmonic perturbation to the free boundary). Thus $v(\xi)$ is at most order $e^{-\xi}$ as $\xi \rightarrow \infty$, implying that $B(\xi)=O\left(\xi e^{-\xi}\right)$ as $\xi \rightarrow \infty$, so $B(\infty)=0$. Also, we must have $|v(0)|<\infty$, so $B(0)=0$ also, and we have two homogeneous boundary conditions on $B$.

Assuming a time-dependence of the form $B(\xi, t)=e^{\mu t} \tilde{B}(\xi)$, we multiply (4.13) by $\tilde{B}(\xi)$ and integrate between zero and infinity, giving

$$
-\int_{0}^{\infty}\left(\tilde{B}^{\prime}(\xi)^{2}\right) d \xi=\frac{\mu}{2} \int_{0}^{\infty} \frac{\tilde{B}(\xi)^{2}}{\xi} d \xi ;
$$

these integrals will be convergent due to the conditions on $B$ at zero and infinity. Thus we deduce that $\mu<0$, and this flat-front travelling wave is a linearly stable solution of the ZST model. 
The reversed flat-front solution (Figure 3 with all arrows reversed) is given in the notation of (3.6) by $F(\zeta, t)=2 t+\zeta, W(\zeta)=-\zeta^{2}$. Exactly the same procedure reveals (4.12) and (4.13) to be replaced by

$$
\frac{\partial}{\partial \zeta}\left(\zeta^{2} \frac{\partial v}{\partial \zeta}\right)+\frac{\zeta}{2} \frac{\partial v}{\partial t}=0, \quad-\frac{\partial B}{\partial t}=2 \xi \frac{\partial^{2} B}{\partial \xi^{2}}, \quad \xi \geqslant 0
$$

respectively, thus in (4.14) the sign of $\mu$ is reversed, and this flat front travelling wave is linearly unstable.

With these two results together, we could have predicted the result for the circular void, since they imply that the rear of the circular void ought to be stable, while the front should be unstable, exactly as we found. We note that the stability of the circular travelling wave when $\sigma \neq 0$ was considered numerically for the full problem by [17, 32] and it was found to be stable for all values of $\sigma$ treated. The linear stability for $\sigma>0$, and the nonlinear stability for $\sigma>0$, remain as interesting unresolved issues.

\subsubsection{The circular void is the only travelling wave void of finite area in the unbounded geometry}

We can in fact prove that the only travelling wave void solution (subject to the asymptotic behaviour (3.2)) having finite void area in an unbounded metal domain is the circular solution of $\S$ 4.1.1. The proof uses the formulation of equation (4.1) as well as the conformal mapping ideas introduced in § 3.1, and relies on a theorem due to Millar [20] (and in part due to Shapiro [26]). The theorem states that for a simple closed analytic curve $\Gamma$, whose Schwarz function $g$ is analytic outside $\Gamma$ and has behaviour $g=O\left(|z|^{N}\right)$ as $|z| \rightarrow \infty$, the following holds: (i) if $N \in\{-2,-3,-4, \ldots\}$ then no admissible $\Gamma$ exists; (ii) if $N=-1$ then $\Gamma$ is a circle centred on the origin; (iii) if $N=0$ then $\Gamma$ is a circle with centre displaced from the origin, and; (iv) if $N=1$ then $\Gamma$ is an ellipse with nonzero eccentricity.

We observe that the right-hand side of (4.1) is analytic throughout the metal domain $\Omega$, and approaches the constant value $2 i$ at infinity. Hence the left-hand side must also represent a function analytic on $\Omega$ with this far field behaviour. We claim that this means $g_{0}(\hat{z})$ is necessarily analytic on the metal domain $\Omega \subset \mathbb{C}$ (except possibly at infinity), so that Millar and Shapiro's theorem is applicable. Once this claim is proved, the result follows quickly, as it is easily seen that the only possible large- $|\hat{z}|$ behaviour of $g_{0}$ in (4.1) compatible with (3.2) is

$$
g_{0}(\hat{z}) \sim \frac{4}{\bar{V}^{2} \hat{z}} \quad \text { as }|\hat{z}| \rightarrow \infty .
$$

Thus, we have case (ii) of the theorem, and we conclude that the void boundary must be a circle centred on the origin, proving the result.

We now prove the claim that $g_{0}(\hat{z})$ must be analytic on the metal domain $\Omega$ if (4.1) holds. This is done in several stages. First, we note that zeros of $g_{0}^{\prime}(\hat{z})$ within $\Omega$ are not allowed, except at infinity where the behaviour is found from (4.16). For, if $g_{0}^{\prime}(\hat{z})$ vanishes at some point, then $\sqrt{g_{0}^{\prime}(\hat{z})}\left(\bar{V} z-V g_{0}(\hat{z})\right)=0$ on the left-hand side of (4.1), while the remaining term $2 \sigma\left(d^{2} / d \hat{z}^{2}\right)\left(1 / \sqrt{g_{0}^{\prime}(\hat{z})}\right)$ is unbounded, giving a singularity of $d w / d \hat{z}$ at that point, hence a contradiction. So $g_{0}^{\prime}(\hat{z})$ cannot vanish at finite points in $\Omega$. 
In terms of the conformal mapping from the unit disc onto the domain $\Omega, g_{0}^{\prime}(\hat{z})$ may be represented as a function of $\zeta$ (see (3.3)):

$$
g_{0}^{\prime}(\hat{z})=-\frac{\bar{f}^{\prime}(1 / \zeta)}{\zeta^{2} f^{\prime}(\zeta)} .
$$

It follows that $\bar{f}^{\prime}(1 / \zeta)$ cannot vanish on $|\zeta| \leqslant 1$ except at $\zeta=0$ (which maps to the point at infinity in $\Omega$ ), and therefore that $f^{\prime}(\zeta)$ cannot vanish on $|\zeta| \geqslant 1$ except at $\zeta=\infty$. By conformality, $f^{\prime}(\zeta) \neq 0$ on $|\zeta| \leqslant 1$, and we conclude that $f^{\prime}(\zeta) \neq 0$ for $0 \leqslant|\zeta|<\infty$. Likewise, $\bar{f}^{\prime}(1 / \zeta) \neq 0$ for $0<|\zeta| \leqslant \infty$. From (4.17) then, $g_{0}^{\prime}(\hat{z})$ is bounded and nonzero (as a function of $\zeta$ ) on the unit disc, except at $\zeta=0$; hence it is bounded and nonzero (as a function of $\hat{z}$ ) on $\Omega$ except at $\hat{z}=\infty$, where the behaviour is given by (4.16). So $g_{0}^{\prime}(\hat{z})$ is bounded on $\Omega$, and nonzero except at infinity.

It follows that $g_{0}^{\prime}(\hat{z})$ can only have certain types of branch-point singularities in $\Omega$ (things like poles would obviously give infinities). Suppose there is a singularity at $\hat{z}=\hat{z}_{0} \in \Omega$; we write

$$
g_{0}^{\prime}(\hat{z})=g_{0}^{\prime}\left(\hat{z}_{0}\right)+S^{\prime}(\hat{z})+\cdots \quad \text { near } \hat{z}=\hat{z}_{0},
$$

where $g_{0}^{\prime}\left(\hat{z}_{0}\right) \neq 0$, and $S^{\prime}(\hat{z}) \rightarrow 0$ as $\hat{z} \rightarrow \hat{z}_{0} . S^{\prime}(\hat{z})$ represents the local singular behaviour, which could be something like $S^{\prime}(\hat{z}) \sim \sqrt{\hat{z}-\hat{z}_{0}}$, or $S^{\prime}(\hat{z}) \sim\left(\hat{z}-\hat{z}_{0}\right) \log \left(\hat{z}-\hat{z}_{0}\right)$, for example. Substituting this behaviour into (4.1) we find

$$
\begin{array}{r}
\left(g^{\prime}\left(\hat{z}_{0}\right)^{1 / 2}+\frac{S^{\prime}(\hat{z})}{2 g^{\prime}\left(\hat{z}_{0}\right)^{1 / 2}}+\cdots\right)\left(z_{0}\left(\bar{V}-V g^{\prime}\left(\hat{z}_{0}\right)\right)-V S(\hat{z})+\cdots\right)- \\
\frac{\sigma}{g^{\prime}\left(\hat{z}_{0}\right)^{3 / 2}} S^{\prime \prime \prime}(\hat{z})=\text { (regular). }
\end{array}
$$

Absorbing the regular parts into the right-hand side we require

$$
-V g^{\prime}\left(\hat{z}_{0}\right)^{1 / 2} S(\hat{z})+\frac{\hat{z}_{0} S^{\prime}(\hat{z})}{2 g^{\prime}\left(\hat{z}_{0}\right)^{1 / 2}}\left(\bar{V}-V g^{\prime}\left(\hat{z}_{0}\right)\right)-\frac{V S(\hat{z}) S^{\prime}(\hat{z})}{2 g^{\prime}\left(\hat{z}_{0}\right)^{1 / 2}}-\frac{\sigma S^{\prime \prime \prime}(\hat{z})}{g^{\prime}\left(\hat{z}_{0}\right)^{3 / 2}} \approx 0,
$$

near $\hat{z}=\hat{z}_{0}$. Considering the different possible leading-order balances in (4.18), it is clear that $S^{\prime \prime \prime}$ balancing $S^{\prime}$ and $S$ leads to trivial nonsingular solutions. In any case, $S^{\prime \prime \prime}$ must be more singular than either $S$ or $S^{\prime}$, if $S$ is itself singular at $\hat{z}_{0}$. So suppose

$$
V S(\hat{z}) S^{\prime}(\hat{z})+\frac{2 \sigma S^{\prime \prime \prime}(\hat{z})}{g^{\prime}\left(\hat{z}_{0}\right)} \approx 0 .
$$

This may be integrated twice, giving

$$
\frac{\sigma S^{\prime}(\hat{z})^{2}}{g^{\prime}\left(\hat{z}_{0}\right)}+\frac{V S(\hat{z})^{3}}{6} \approx k_{1} S(\hat{z})+k_{2},
$$

for arbitrary $k_{1}$ and $k_{2}$. The only possible singular behaviour of solutions to this equation is easily checked to be $S(\hat{z})=O\left(\hat{z}-\hat{z}_{0}\right)^{-2}$, but since this represents an unbounded singularity in $g^{\prime}(\hat{z})$, it is not allowable.

We conclude that there are no allowable singularities of $g^{\prime}(\hat{z})$ within $\Omega$, hence $g^{\prime}(\hat{z})$, and therefore $g(\hat{z})$, must be analytic within $\Omega$. This proves our claim, which gives the result.

We remark that these arguments are easily applied also to the ZST problem $\sigma=0$, so in this case also the only travelling-wave solution of finite void area is the circular 
void. However, they do not work when $V=0$ (static solutions), in which case there are non-circular solutions: see $\S 4.2$ below.

Taken together with the linear stability of the NZST travelling-wave circle problem (claimed from the numerical results of Mahadevan \& Bradley [17] and Wang et al. [32]), this is an important result, as it suggests that for sufficiently large values of the surface tension parameter $\sigma$, a small void moving within a conductor will approach this travellingwave circular form. As remarked in $\S 4.1 .3$ though, since the ZST problem is linearly unstable we expect the threshold perturbation (below which perturbations will decay, but above which they may grow) to tend to zero as $\sigma \rightarrow 0$. Thus for small values of $\sigma$, instabilities may be manifested, and this travelling-wave form may not be approached.

We note here that if the assumption of a perfectly insulating void is relaxed, i.e. the electrical conductivity within the void is nonzero (though always less than that outside the void), then other travelling wave solutions may exist. The model in this case is more complicated, as Laplace's equation must be solved inside the void as well as outside. Here the single boundary condition $\partial \phi / \partial n=0$ on $\partial \Omega$ is replaced by two jump conditions:

$$
[\phi]_{\partial \Omega}=0, \quad\left[\varrho \frac{\partial \phi}{\partial n}\right]_{\partial \Omega}=0,
$$

where $\varrho$ represents the electrical conductivity inside or outside the void. The second of these conditions represents continuity of the current passing through the void boundary (no jump in $\boldsymbol{J} \cdot \boldsymbol{n}$ ), while the continuity of $\phi$ follows from the fact that $\nabla \wedge \boldsymbol{E} \equiv-\partial \boldsymbol{B} / \partial t$ must be bounded at the void boundary. Wang et al. [32] consider the case in which $\varrho_{\text {void }}=\varrho_{\text {metal }}$, so that the electric field is simply uniform throughout. In this case their numerical simulations indicate that an initially circular void can approach an egg-shaped travelling wave ([32], Figure 4).

\subsection{Steady solutions}

It is of interest to note that the steady model, with $v_{n} \equiv 0$ in (2.3), is exactly the steady one-phase Hele-Shaw model with surface tension effects included at the free boundary, if the electric potential $\phi$ is identified with $p$, the pressure in the fluid, the metal domain $\Omega$ with the domain occupied by fluid, and the void with an air-bubble in the cell. In the time-dependent Hele-Shaw problem, $p$ is harmonic within the fluid domain $\Omega$, and satisfies the kinematic boundary condition $\partial p / \partial n=-v_{n}$, and the dynamic boundary condition $p=-\sigma \kappa$, using the same notation as our formulation (2.7)-(2.9). Thus if one considers the steady case of each problem, with $v_{n}=0$, the Hele-Shaw kinematic boundary condition is equivalent to the 'insulating void' condition (2.8), and the electromigration kinematic boundary condition (2.9) may be integrated twice with respect to arclength $s$ along the boundary:

$$
\phi=-\sigma \kappa+k_{1} s+k_{2} .
$$

For single-valuedness of the potential, $k_{1}$ must be zero, and the arbitrary constant $k_{2}$ may be absorbed into $\phi$, so can be taken to be zero without loss of generality. Hence (4.19) is equivalent to the Hele-Shaw dynamic boundary condition.

A family of exact solutions for the steady problem of a finite bubble in the cell was 


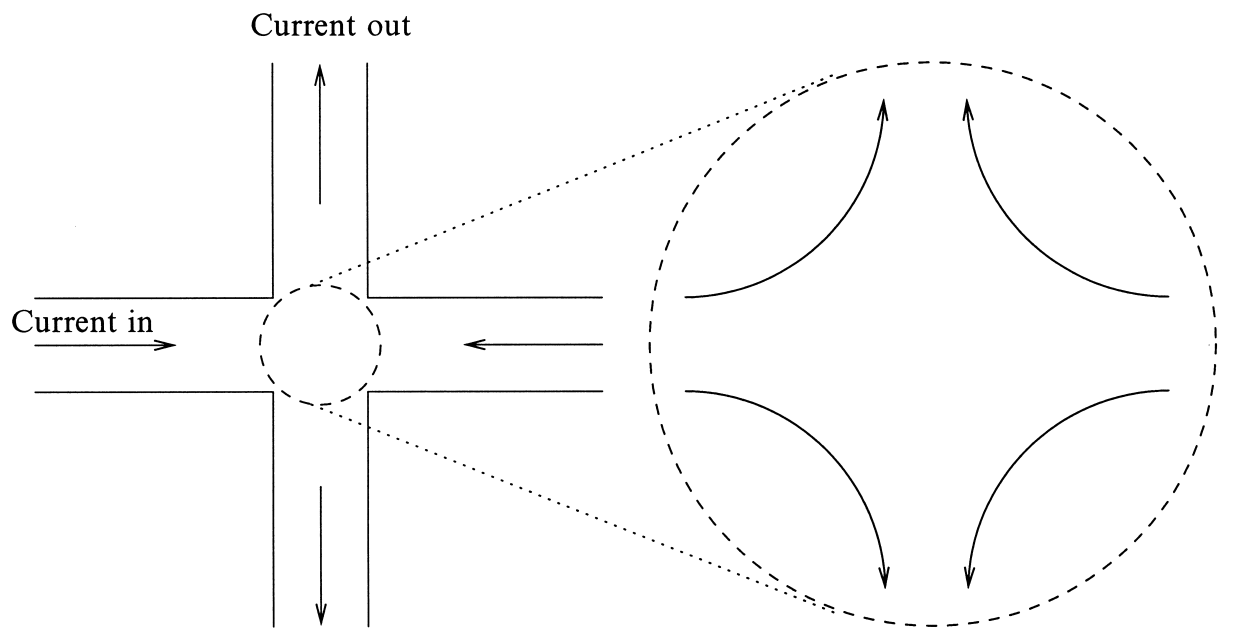

Arrows indicate direction of electric field

FIGURE 4. The local situation at the junction of two conductors.

discovered by Entov et al. [8], with appropriate behaviour at infinity; it follows that these are also solutions to our model, provided $w(z)$ has the correct behaviour at infinity. In the Hele-Shaw context, the solutions of Entov et al. [8] involve a bubble symmetric about both $x$ - and $y$-axes, with the complex potential $\mathscr{F}(z)(p=\Re(\mathscr{F}))$ having the behaviour

$$
\mathscr{F}(z)=\frac{m}{2 \pi} z^{n}+\{\text { analytic part }\} \quad \text { as }|z| \rightarrow \infty,
$$

where $m>0$ measures the strength of the 'multipole singularity' at infinity. For our problem $\mathscr{F} \equiv w$, so the complex electric potential has the behaviour (4.20) at infinity. The case $n=1$, which is our usual far-field condition, has no solution, but values $n>1$ give nontrivial equilibrium shapes. Consider for example the case $n=2$. This far-field behaviour corresponds to an electric field aligned along hyperbolae at infinity, since the 'streamfunction' $\psi$ (the harmonic conjugate of $\phi$ has behaviour $\psi \sim m x y / \pi$ for $x^{2}+y^{2} \gg 1$. Such behaviour may be appropriate to describe the local situation at a junction of two conductors, where current is flowing as indicated in Figure 4.

For each value of $n \geqslant 2$ it is found that, depending on the value of the dimensionless parameter $\mu=(2 \pi \sigma(n+1) / m)^{-2 /(n+1)} S / \pi$ (where $S$ is the cross-sectional area of the bubble/void), there are either two, one or no solutions to the problem. More precisely, for $\mu<\mu^{*}(n)$ (sufficiently small voids) there are two solutions; for $\mu=\mu^{*}(n)$ there is one solution (critical void size), and for $\mu>\mu^{*}(n)$ there are no solutions. The values of $\mu^{*}(n)$ and the possible equilibrium shapes are given in Entov et al. [8]. Thus we conclude that small voids can exist in equilibrium at the junction between two (or more) conductors, as indicated in figure 4. For this scenario the two equilibrium voids are one that is rather elliptical in shape, and one that is peanut-shaped, both elongated along the $x$-axis. It is conjectured in Entov et al. [8] that where only one equilibrium shape exists, it is stable, and where two exist, one is stable and the other is not, conclusions which would follow through for the void solutions; however the stability analysis is not carried out. 


\section{The strip geometry: Voids in the interior}

Ideally, we would like to be able to find some exact (travelling-wave or otherwise) solutions in the strip geometry, such as those found numerically for the ZST problem by Ben Amar [2] (infinite fingers, and finite rounded voids, both symmetric travelling-wave shapes). However, this appears far from simple, and we have been unsuccessful so far. As noted in $\S 3$, to deal with the insulating conductor boundaries one has to consider a periodic array of voids. Suppose we wish to use the conformal mapping technique embodied in (3.6) to find an infinite symmetric 'finger' travelling wave solution. If we map from the right-half plane via $z=F(\zeta)$, the map must be periodic in the $y$-direction, generating an infinite array of identical fingers, so in the $\zeta$-plane the insulating walls must be the image of lines $\mathfrak{I}(\zeta)=$ constant; see Figure 5 . Consideration of the map far ahead of the fingers $(\mathfrak{R}(z) \rightarrow+\infty, \mathfrak{R}(\zeta) \rightarrow+\infty)$ shows that $F(\zeta) \sim \zeta$ there (or some constant multiple). Thus the complex potential $W(\zeta)$ in the right-half $\zeta$-plane must satisfy $W(\zeta) \sim \zeta$ as $\mathfrak{R}(\zeta) \rightarrow+\infty$, and have constant imaginary part on those lines $\mathfrak{I}(\zeta)=$ constant which correspond to the insulating walls. It follows that for a symmetric finger

$$
W(\zeta)=\frac{2}{\pi} \log (\cosh (\pi \zeta / 2)),
$$

since this is periodic with $\mathfrak{I}(W)= \pm 1$ on the lines $\mathfrak{I}(\zeta)= \pm 1$ (which correspond to the insulating sides of the strip), and has zero imaginary part on $\mathfrak{R}(\zeta)=0$ (which gives the void boundary). Also, (5.1) has the correct far-field behaviour as $\mathfrak{R}(\zeta) \rightarrow+\infty$. Thus, neglecting surface tension effects, (3.6) reduces to the problem of finding the appropriate conformal map $F(\zeta, t)$ satisfying

$$
F_{t}(\zeta) \bar{F}^{\prime}(-\zeta)+\bar{F}_{t}(-\zeta) F^{\prime}(\zeta)=-2 \frac{\partial}{\partial \zeta}\left[\frac{\tanh (\pi \zeta / 2)}{\left(F^{\prime}(\zeta) \bar{F}^{\prime}(-\zeta)\right)^{1 / 2}}\right]
$$

together with the appropriate behaviour far ahead of, far behind, and at the tip of, the finger. If one assumes a travelling-wave form for $F, F(\zeta, t)=b(t)+F_{0}(\zeta)$, one can integrate (5.2) (the constant of integration has to be zero from considerations at the finger-tip $z=b(t)$ where $F_{0}$ has the local behaviour $\left.F_{0}(\zeta) \sim-\zeta(\pi /(2 \dot{b}))^{1 / 2}\right)$ giving

$$
F_{0}(\zeta)-\bar{F}_{0}(-\zeta)=-\frac{2}{\dot{b}} \frac{\tanh (\pi \zeta / 2)}{\left(F_{0}^{\prime}(\zeta) \bar{F}_{0}^{\prime}(-\zeta)\right)^{1 / 2}},
$$

but the problem is still formidable, and (other than the numerical evidence of Ben Amar [2]) we have no guarantee that a solution $F_{0}(\zeta)$ satisfying all the requirements exists. The numerical finger solution appears to be uniquely selected, with a calculated width of 0.666 times the channel width, suggesting that the true width $\lambda$ is $2 / 3$. Even this unique selection is still a puzzle, as asymptotic analysis far behind the finger-tip gives only the single relation $\dot{b} \lambda(1-\lambda)=-1$, and it is not clear how to obtain a second relation. We remark that the finger-shape which solves the zero surface tension Hele-Shaw channel fingering problem [23] does not provide a solution to the electromigration problem, for any value of the finger width $\lambda$. Hele-Shaw $\lambda=2 / 3$ fingers are much blunter than those calculated in Ben Amar [2] for this problem.

Seeking exact finite-void solutions within the strip geometry, one encounters similar difficulties. Voids at the edge of a conducting strip (for example, see Figure 1) are possibly more realistic from an experimental point of view, but exact solutions are again elusive 

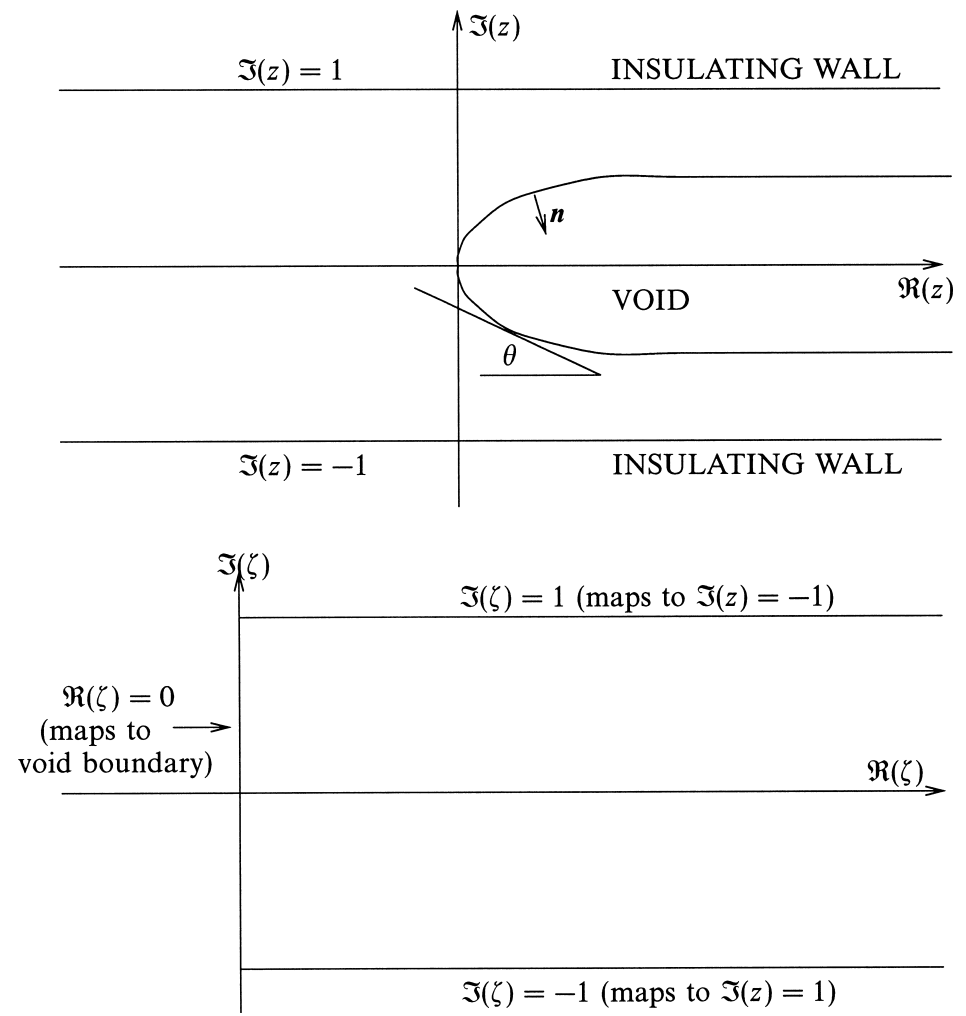

FIGURE 5. The correspondence between $z$ - and $\zeta$-planes for a finger-shaped void.

(and in fact we cannot hypothesise a simple periodic mapping in this case when the void is not enclosed by metal). We use asymptotic analysis to consider this situation in some detail in $\S 6$.

Being unable to find exact solutions in the strip geometry, we turn to approximate solutions, using methods from asymptotics.

\subsection{Small voids in a strip}

Throughout this subsection we neglect the effect of surface tension on the void shape and motion. Consider the simple case of a small, approximately circular void, radius $\epsilon h$, moving in a strip of width $2 h$, where $\epsilon \ll 1$. We assume the dimensionless model (2.7)-(2.10), where the lengthscale $l$ used to nondimensionalise (as described in $\S 2$ ) is taken to be $h$ : the insulating boundaries are then at $y= \pm 1$, and the void has radius $\epsilon$. These dimensionless variables are the 'outer' variables, in which the void appears as a point singularity in the electric field, which, far from the singularity, is $w(z) \sim z$.

If $z=b(t)=\alpha(t)+i \beta(t)$ denotes the position of the void in the outer variables, then we work with the moving coordinate $\hat{z}=z-b(t)$ in which the void is situated at the origin. We define the inner variable $\tilde{z}=\tilde{x}+i \tilde{y}$ by $\hat{z}=\epsilon \tilde{z}$ (in general, a tilde denotes an 'inner' quantity). At leading order in the inner, the void cannot 'feel' the conductor walls, and 
the solution is a circular void of unit radius, exactly as given in $\S$ 4.1.1:

$$
\tilde{w}_{0}(\tilde{z})=\frac{\tilde{V}}{2}\left(\tilde{z}+\frac{1}{\tilde{z}}\right), \quad \tilde{g}_{0}(\tilde{z})=\frac{1}{\tilde{z}},
$$

where $\tilde{V}$ is to be determined. This must match [31] onto the leading order outer solution as $\tilde{z} \rightarrow \infty$. Writing (5.4) in terms of the outer variable we find

$$
\tilde{w}_{0}(\hat{z})=\frac{\tilde{V}}{2 \epsilon}\left(\hat{z}+\frac{\epsilon^{2}}{\hat{z}}\right),
$$

thus matching requires $\tilde{V}=2 \epsilon$, and the void travels with dimensionless speed 2 in the negative $x$-direction. The other piece of information we obtain from (5.5) is the nature of the singularity induced by the void in the outer solution. Together with the condition that $\mathfrak{I}(w)$ be constant on the insulated walls $y= \pm 1(\hat{y}= \pm 1-\beta)$, this enables us to write down the next term in the outer solution:

$$
\begin{aligned}
w(\hat{z}) & =\hat{z}+\epsilon^{2} \frac{\pi}{4}\left(\operatorname{coth}\left(\frac{\pi}{4} \hat{z}\right)+\tanh \left(\frac{\pi}{4}(\hat{z}+2 i \beta)\right)\right)+o\left(\epsilon^{2}\right) \\
\Rightarrow & \frac{d w}{d \hat{z}}=1-\epsilon^{2} \frac{\pi^{2}}{16}\left(\operatorname{cosech}^{2}\left(\frac{\pi}{4} \hat{z}\right)-\operatorname{sech}^{2}\left(\frac{\pi}{4}(\hat{z}+2 i \beta)\right)\right)+o\left(\epsilon^{2}\right) .
\end{aligned}
$$

To examine next order in the inner solution, we expand (5.7) near $\hat{z}=0$, and write it in terms of the inner variables to see how the expansion for $d \tilde{w} / d \tilde{z}$ proceeds, and what the large- $\tilde{z}$ behaviour must be. Using the fact that $\operatorname{cosech} x \sim(1 / x)-x / 6+O\left(x^{3}\right)$ for small $x$ we see that (5.7) implies the large $|\tilde{z}|$ behaviour

$$
\frac{1}{\epsilon} \frac{d \tilde{w}}{d \tilde{z}}=1-\frac{1}{\tilde{z}^{2}}+\epsilon^{2} \frac{\pi^{2}}{16}\left(\frac{1}{3}+\sec ^{2}\left(\frac{\pi \beta}{2}\right)\right)+O\left(\epsilon^{3}\right) \equiv 1-\frac{1}{\tilde{z}^{2}}+\epsilon^{2} \lambda+O\left(\epsilon^{3}\right)
$$

(defining the real constant $\lambda$ for convenience). Thus the next term in the expansion for $\tilde{w}$ is $O\left(\epsilon^{3}\right)$. The expansions in the inner proceed as:

$$
\begin{array}{r}
\tilde{w}=\epsilon\left(\tilde{z}+\frac{1}{\tilde{z}}+\epsilon^{2} \tilde{w}_{2}+\epsilon^{3} \tilde{w}_{3}+\cdots\right), \\
\tilde{g}=\frac{1}{\tilde{z}}+\epsilon^{2} \tilde{g}_{2}+\epsilon^{3} \tilde{g}_{3}+\cdots, \\
\tilde{V}=\epsilon\left(2+\epsilon^{2} \tilde{V}_{2}+\epsilon^{3} \tilde{V}_{3}+\cdots\right),
\end{array}
$$

where $\tilde{w}_{2}$ has the far-field behaviour (necessary for the matching)

$$
\tilde{w}_{2}^{\prime}(\tilde{z}) \sim \lambda
$$

as $|\tilde{z}| \rightarrow \infty$. Substituting these expansions into (4.1) (with $V=\tilde{V}, w=\tilde{w}, g=\tilde{g}, z=\tilde{z}$ and $\sigma=0$ ), leading order matching is now automatic, and at first order we obtain

$$
\left(\tilde{z}^{2}-1\right) \frac{d \tilde{g}_{2}}{d \tilde{z}}+\frac{2}{\tilde{z}} \tilde{g}_{2}=-2 \frac{d \tilde{w}_{2}}{d \tilde{z}}+\overline{\tilde{V}}_{2}-\frac{\tilde{V}_{2}}{\tilde{z}^{2}} .
$$

We require conservation of the area $S$ of the perturbed void, which may be expressed as

$$
S=\iint_{\text {Void }} d x d y=\frac{1}{2 i} \oint \overline{\tilde{z}} d \tilde{z}=\frac{1}{2 i} \oint \tilde{g}(\tilde{z}) d \tilde{z}=\frac{1}{2 i} \oint \tilde{g}_{0}(\tilde{z}) d \tilde{z},
$$

where the line integrals are taken around the void boundary in the anticlockwise direction. 
Hence we require

$$
\oint \tilde{g}_{m}(\tilde{z}) d \tilde{z}=0, \quad m>0 .
$$

Using (5.10) and (5.9) we find the most general solution for the first order problem is

$$
\tilde{w}_{2}(\tilde{z})=\lambda\left(\tilde{z}+\frac{1}{\tilde{z}}\right)+\frac{c_{2}}{\tilde{z}^{2}}, \quad \tilde{g}_{2}(\tilde{z})=c_{2}\left(1+\frac{1}{\tilde{z}^{2}}\right), \quad \tilde{V}_{2}=2 \lambda,
$$

where $c_{2}$ is an arbitrary constant. The void is still circular at this order, with at most an order $\epsilon^{2}$ shift in its centre along the direction of its motion; without loss of generality (by absorbing this shift into the leading order solution) we may take $c_{2}=0$ and so $\tilde{g}_{2}(\tilde{z}) \equiv 0$. The only effect of the finite conductor width at this order is the real $O\left(\epsilon^{2}\right)$ correction to its velocity provided by $\tilde{V}_{2}$ : the void velocity in the (dimensionless) outer variables is $V=$ $2+2 \epsilon^{2} \lambda$. Any correction to the void shape comes in at a higher order. This is in agreement with the numerical results of Ben Amar [2], who found that voids translating in the interior of a conducting strip are very nearly circular in shape, even when relatively large.

\section{The strip geometry: Voids at a conductor boundary}

In this section we abandon our complex variable approach, and instead use asymptotic methods similar to those used in hydrodynamics to describe free-surface disturbances on water of finite depth (see e.g. [22] chapters 2 and 3 for a discussion). We return to the dimensional problem (2.1)-(2.4), writing $A=-Z^{*} e M$ and $B=M \omega \gamma$ (both positive) for convenience. The problem is shown in figure 6 , with a void on the upper boundary which for the moment is assumed to be of infinite extent, and to be described by $y=\eta(x, t)$, with $\eta(\infty, t)=0$ and $\eta(-\infty, t)=\mathscr{2}$.

With this notation we have

$$
v_{n}=\frac{\eta_{t}}{\left(1+\eta_{x}^{2}\right)^{1 / 2}}, \quad \kappa=\frac{\eta_{x x}}{\left(1+\eta_{x}^{2}\right)^{3 / 2}},
$$

and the derivative with respect to arclength $s$ along the free boundary is given by

$$
\partial_{s}=\frac{1}{\left(1+\eta_{x}^{2}\right)^{1 / 2}}\left(\partial_{x}+\eta_{x} \partial_{y}\right)
$$

thus the boundary conditions may be expressed in terms of $\phi$ and $\eta$. The full dimensional model is:

$$
\begin{aligned}
& \nabla^{2} \phi=0 \quad-h_{0}<y<\eta, \\
& \phi_{y}=\eta_{x} \phi_{x} \quad \text { on } y=\eta \text {, } \\
& -\eta_{t}=A\left(\partial_{x}+\eta_{x} \partial_{y}\right)\left(\frac{\phi_{x}+\eta_{x} \phi_{y}}{\left(1+\eta_{x}^{2}\right)^{1 / 2}}\right)+B \frac{\partial}{\partial x}\left\{\frac{1}{\left(1+\eta_{x}^{2}\right)^{1 / 2}} \frac{\partial}{\partial x}\left(\frac{\eta_{x x}}{\left(1+\eta_{x}^{2}\right)^{3 / 2}}\right)\right\} \\
& \text { on } y=\eta \text {, } \\
& \phi_{y}=0 \quad \text { on } y=-h_{0}, \\
& \phi_{x} \sim E_{\infty}, \quad \eta \sim 0 \quad \text { as } x \rightarrow \infty, \\
& \phi_{x} \sim \frac{h_{0} E_{\infty}}{h_{0}+\mathscr{2}}, \quad \eta \sim \mathscr{2} \quad \text { as } x \rightarrow-\infty .
\end{aligned}
$$




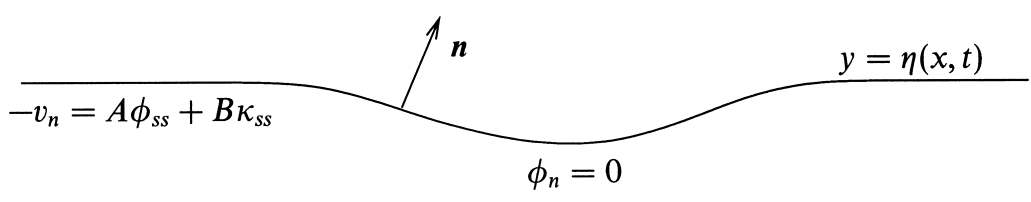

METAL FILM

Direction of $\Omega$

electric field

$$
\nabla^{2} \phi=0
$$

\begin{tabular}{lll}
\hline CONDUCTOR BOUNDARY $\quad \phi_{n}=0$ & $y=-h_{0}$
\end{tabular}

FIGURE 6. The full problem in the dimensional variables.

Suppose now that the initial profile is of the form $\eta(x, 0)=\eta_{0} N(x / l)$, so that $l$ and $\eta_{0}$ are lengthscales of the disturbance in the $x$ and $y$ directions, respectively. We then scale:

$$
x=l \bar{x}, \quad y=h_{0} \bar{y}, \quad \eta=\eta_{0} \bar{\eta}, \quad t=t_{0} \bar{t}, \quad \phi=\phi_{0} \bar{\phi},
$$

where $t_{0}$ and $\phi_{0}$ are to be determined. It is natural to scale $\phi$ with the applied electric field at infinity; we thus choose $\phi_{0}=E_{\infty} l$. The appropriate time scaling is obtained from equation (6.3), and clearly depends upon which of the two terms on the right-hand side of this equation dominate. If the second term dominates we have, at least to leading order, motion of the free surface under surface tension subject to a volume constraint. Since this has been extensively studied elsewhere (see Elliott \& Garcke [7] and the references therein), and is not really the subject of this work, we scale time with the first of the two terms by taking $t_{0}=l \eta_{0} /\left(A E_{\infty}\right)$. We thereby implicitly assume that effects due to the electric field either dominate, or are comparable with, surface tension effects. Before writing down the dimensionless model it is helpful to introduce the following two dimensionless parameters:

$$
\epsilon=\frac{\eta_{0}}{h_{0}} \sim \frac{\text { 'amplitude' }}{\text { 'depth' }}, \quad \delta=\frac{h_{0}}{l} \sim \frac{\text { 'depth' }}{\text { 'wavelength' }}
$$

(borrowing concepts from fluid dynamics rather loosely here). With these definitions the rescaling discussed above yields the following dimensionless model (dropping overbars for convenience):

$$
\begin{aligned}
& \delta^{2} \phi_{x x}+\phi_{y y}= 0 \quad-1<y<\epsilon \eta \\
& \phi_{y}= \epsilon \delta^{2} \eta_{x} \phi_{x} \quad \text { on } y=\epsilon \eta, \\
&-\eta_{t}=\left(\partial_{x}+\epsilon \eta_{x} \partial_{y}\right)\left(\frac{\phi_{x}+\epsilon \eta_{x} \phi_{y}}{\left(1+\epsilon^{2} \delta^{2} \eta_{x}^{2}\right)^{1 / 2}}\right) \\
&+\sigma^{*} \frac{\partial}{\partial x}\left\{\frac{1}{\left(1+\epsilon^{2} \delta^{2} \eta_{x}^{2}\right)^{1 / 2}} \frac{\partial}{\partial x}\left(\frac{\eta_{x x}}{\left(1+\epsilon^{2} \delta^{2} \eta_{x}^{2}\right)^{3 / 2}}\right)\right\} \quad \text { on } y=\epsilon \eta, \\
& \phi_{y}= 0 \quad \text { on } y=-1, \\
& \phi_{x} \sim 1, \quad \eta \sim 0 \quad \text { as } x \rightarrow \infty
\end{aligned}
$$




$$
\phi_{x} \sim \frac{1}{1+\epsilon \mathscr{Q}^{*}}, \quad \eta \sim \mathscr{2}^{*} \quad \text { as } x \rightarrow-\infty,
$$

where dimensionless coefficients $\mathscr{Q}^{*}$ and $\sigma^{*}$ are defined as follows:

$$
\mathscr{2}^{*}=\frac{\mathscr{Q}}{\eta_{0}} \quad \sigma^{*}=\frac{B \eta_{0}}{A l^{3} E_{\infty}} \quad\left(\sigma^{*} \leqslant O(1)\right) .
$$

To get a simplified approximate model, we want to consider the case in which one or both of the parameters $\epsilon$ and $\delta$ are small. We now consider different cases.

The case $\delta \ll 1, \epsilon=1$

This is the case of a long-wavelength but deep void. Here we search for an asymptotic solution of the form

$$
\phi=\phi^{(0)}+\delta^{2} \phi^{(1)}+O\left(\delta^{4}\right), \quad \eta=\eta^{(0)}+\delta^{2} \eta^{(1)}+O\left(\delta^{4}\right) .
$$

Substitution of the above into equations (6.7)-(6.11) at leading order yields

$$
\begin{aligned}
\phi^{(0)} & =\phi^{(0)}(x, t), \\
\eta_{t}^{(0)} & =\phi_{x x}^{(0)}-\sigma^{*} \eta_{x x x x}^{(0)}, \\
\phi_{x}^{(0)} & \sim 1, \quad \eta^{(0)} \sim 0 \quad \text { as } x \rightarrow \infty \\
\phi_{x}^{(0)} & \sim \frac{1}{1+\mathscr{2}^{*}}, \quad \eta^{(0)} \sim \mathscr{2}^{*} \quad \text { as } x \rightarrow-\infty,
\end{aligned}
$$

whilst at first order we find the following problem for $\phi^{(1)}$ :

$$
\begin{aligned}
\phi_{y y}^{(1)}+\phi_{x x}^{(0)} & =0, \\
\phi_{y}^{(1)} & =0 \quad \text { on } y=-1, \\
\phi_{y}^{(1)} & =\eta_{x}^{(0)} \phi_{x}^{(0)} \quad \text { on } y=-1 .
\end{aligned}
$$

Integrating equation (6.17) between $y=-1$ and $y=\eta^{(0)}$ and applying the boundary conditions (6.18) and (6.19) leads to the following relation:

$$
\frac{\partial}{\partial x}\left(\phi_{x}^{(0)}\left(1+\eta^{(0)}\right)\right)=0
$$

expressing conservation of electric current. We integrate this equation, apply the boundary conditions (6.15) and (6.16) and use the result to eliminate $\phi_{x}^{(0)}$ from (6.14). Writing $f=\eta^{(0)}+1$ this leads to the following parabolic nonlinear PDE describing the evolution of the free surface:

$$
f_{t}+\left(\frac{1}{f}\right)_{x}+\sigma^{*} f_{x x x x}=0, \quad f(\infty, t)=1, \quad f(-\infty, t)=\lambda,
$$

where $\lambda=1+2^{*}$. Thus, we have a model with just one dimensionless parameter $\sigma^{*}$, a kind of dimensionless surface tension coefficient, which measures the relative effects of surface diffusion due to the surface tension and surface diffusion due to the electric field. A simple extension of this model is to consider a variable lower conductor boundary, $y=-h_{0} H(x / l)$ (in the dimensional variables). This leads to the alternative equation for 
the void profile $f=1+\eta$ :

$$
f_{t}+\left(\frac{1}{(f+H)}\right)_{x}+\sigma^{*} f_{x x x x}=0
$$

plus suitable boundary conditions at infinity. However, we shall mostly be concerned with the model (6.21).

The case $\delta=1, \epsilon \ll 1$

This is the case (also considered by Schimschak \& Krug [24]) of a small-amplitude 'disturbance' (the void) at the boundary, whose wavelength is comparable to the conductor width. We look for an asymptotic solution of the form

$$
\phi=\phi^{(0)}+\epsilon \phi^{(1)}+\cdots, \quad \eta=\eta^{(0)}+\epsilon \eta^{(1)}+\cdots .
$$

Substituting these expansions into equations (6.7)-(6.12) we find that $\phi^{(0)} \equiv x$ to leading order. If $\sigma^{*}=O(1)$ consideration of the leading order expansion of equation (6.9) gives the following equation for the free surface

$$
f_{t}=-\sigma^{*} f_{x x x x}
$$

where $f=1+\epsilon \eta^{(0)}$ represents the total height of the film. In other words the evolution is the same as before but without the nonlinear terms arising from the electric field. We thus consider $\sigma^{*}=\epsilon \Sigma$ where $\Sigma=O(1)$. It is clear that the evolution of the free surface takes place over an $O(1 / \epsilon)$ timescale, so we introduce the long timescale $T$ defined by $t=\epsilon T$ and consider equation (6.9) at leading order,

$$
-\eta_{T}^{(0)}=\phi_{x x}^{(1)}+\Sigma \eta_{x x x x}^{(0)} \quad \text { on } y=0,
$$

together with the first order behaviour for $\phi$ obtained from equations (6.7)-(6.8) and (6.10)-(6.12):

$$
\begin{aligned}
\nabla^{2} \phi^{(1)} & =0 \quad-1<y<0, \\
\phi_{y}^{(1)} & =\eta_{x}^{(0)} \quad \text { on } y=0, \\
\phi_{y}^{(1)} & =0 \quad \text { on } y=-1, \\
\phi_{x}^{(1)} & \sim 0 \quad \text { as } x \rightarrow \infty, \quad \phi_{x}^{(1)} \sim-2^{*} \quad \text { as } x \rightarrow-\infty .
\end{aligned}
$$

This can be solved by Fourier transforming in $x$; solutions are of the form

$$
\begin{aligned}
& \hat{\phi}^{(1)}=D(k) \cosh (k(y+1)) e^{i \omega T}, \\
& \hat{\eta}^{(0)}=-i D(k) \sinh (k(y+1)) e^{i \omega T},
\end{aligned}
$$

where $\omega$ satisfies the dispersion relation:

$$
i \omega=-\Sigma k^{4}+i k^{2} \operatorname{coth} k
$$

(this relation was also given in Schimschak \& Krug [24]). Hence for $\Sigma>0$ we see that such solutions are decaying and that voids of this type die away as $t$ increases. 
The case $\delta \ll 1, \epsilon \ll 1$

This is the case of a long wavelength void, which is also shallow relative to the strip width. The equation for $f=\epsilon \eta+1$ may be obtained by a direct analysis like that above, or by noting that $\epsilon \ll 1$ corresponds to $f \approx 1$ in (6.21), hence we obtain the linear equation

$$
f_{t}-f_{x}+\sigma^{*} f_{x x x x}=0, \quad f(\infty, t)=1, \quad f(-\infty, t)=1+\epsilon \mathscr{Q}^{*} .
$$

We give this result only for completeness, and do not consider solutions to this model. Observe that in the ZST limit $\sigma^{*}=0$, we have a trivial linear wave equation so that any initial profile simply propagates without change of shape.

\subsection{Properties of the model (6.21)}

\subsubsection{Voids of finite area conserve their area}

We first show that according to (6.21) voids of finite area conserve their area, which is certainly a desirable feature of any approximate model, since this requirement was built into the original exact model on the grounds of mass conservation. The void area $\mathscr{A}$ is given by

$$
\mathscr{A}=\int_{-\infty}^{\infty}(1-f) d x
$$

wherever this integral converges. Thus

$$
\frac{d \mathscr{A}}{d t}=\frac{d}{d t} \int_{-\infty}^{\infty}(1-f) d x=-\int_{-\infty}^{\infty} f_{t} d x=\left[\frac{1}{f}+\sigma^{*} f_{x x x}\right]_{-\infty}^{\infty} .
$$

The expression on the right-hand side here must be zero, since for a void of finite area we require $f$ to approach the constant value 1 at $x= \pm \infty$, and the derivatives to vanish.

(Note that it is possible for the 'main part' of void to appear to change its area when the conducting strip is infinitely long, as finite changes in the area of the main part may be compensated by infinitesimal changes at infinity.)

\subsubsection{The case of negligible surface tension}

In this case the void motion may be approximated by equation (6.21) with $\sigma^{*}=0$. We then have a first order nonlinear wave equation for $f$,

$$
f_{t}-\frac{1}{f^{2}} f_{x}=0,
$$

for which we can write the solution in implicit form as

$$
f(x, t)=f_{0}\left(x+\frac{t}{f(x, t)^{2}}\right),
$$

where $f_{0}$ is the initial void profile. Solutions are wavelike, with effective wavespeed $1 / f^{2}$, which means that the void profile steepens since the deeper parts of the profile move faster. Hence these solutions exhibit finite-time blow-up, which occurs when $f_{x}$ becomes infinite for some point on the profile. To continue beyond this shock formation we need to rescale 
distance so as to reintroduce the effects of surface tension: $x=\sigma^{* 1 / 3} x^{\prime}, t=\sigma^{* 1 / 3} t^{\prime}$. Two possibilities then exist: either the lengthscale of the rescaled equation is no longer large in comparison to the height of the film, and the long-wavelength theory breaks down; or it is still large in comparison to the height. In the former case, the subsequent behaviour is described by the full problem. In the latter case, the small-scale behaviour about the shock is described by equation (6.21) with $\sigma^{*}=1$ and, in the light of $\S 6.1 .4$, we expect that this can be approximated by a travelling wave solution with speed $c=1 /\left(f^{+} f^{-}\right)$, where $f^{+}$and $f^{-}$are the values of $f$ on either side of the shock. The speed of the shock will of course also be $c$.

Despite the general formula in (6.25), it is difficult to find closed-form solutions for a realistic initial profile $f_{0}$. Consider for example 'compact support' void solutions, for which the initial void lies along $(-1,1)$. Since the wave-speed of solutions is $-1 / f^{2}$, and $f \equiv 1$ outside the void, the support for $t>0$ is $(-1-t, 1-t)$. Unless we impose the condition that all derivatives of $f$ vanish at the edge of the support such solutions must be of non-smooth shape, but when considering the ZST problem $\sigma^{*}=0$ this is not so important; we may think of them as being 'weak solutions' to the model.

Even with such a simple initial void shape as a parabola, the implicit equation we have to solve for $f$ is a quintic polynomial and must be solved numerically. However, there is one initial profile for which we can solve explicitly: this is the initially 'pointy' profile given by

$$
f_{0}(x)= \begin{cases}1 & |x|>1 \\ \sqrt{\lambda^{2}-x\left(1-\lambda^{2}\right)} & -1<x<0 \\ \sqrt{\lambda^{2}+x\left(1-\lambda^{2}\right)} & 0<x<1 .\end{cases}
$$

For $t>0$ the profile is still in three parts: $-1-t<x<s(t), s(t)<x<1-t$, where $s(t)$ is the position of the pointy tip; and the remainder of the $x$-axis, for which $f \equiv 1$. To find the void profile for $t>0$ we have to solve the following equation for $f$ :

$$
f=f_{0}\left(x+\frac{t}{f^{2}}\right)=\sqrt{\lambda^{2} \pm\left(1-\lambda^{2}\right)\left(x+\frac{t}{f^{2}}\right)}
$$

(which reduces to a quadratic for $f^{2}$ ) on the appropriate regions. The position of the tip $s(t)$ is then determined by the condition that the two parts of the profile meet up at this point. We find (choosing the sign of the square-root consistent with the initial data):

$$
\begin{gathered}
f(x, t)=\frac{1}{\sqrt{2}}\left(\lambda^{2} \pm x\left(1-\lambda^{2}\right)+\left[\left(\lambda^{2} \pm x\left(1-\lambda^{2}\right)\right)^{2} \pm 4 t\left(1-\lambda^{2}\right)\right]^{1 / 2}\right)^{1 / 2} \\
s(t)=-\frac{t}{\lambda^{2}} .
\end{gathered}
$$

Thus the deepest point of the void travels with constant speed, which means (since void speed $=1 / f^{2}$ ) that the void maintains a constant depth. Blow-up occurs when $f_{x}$ first becomes infinite, which for this case happens at the point $x=s(t)^{-}$, at time $t^{*}=\lambda^{4} /\left(1-\lambda^{2}\right)$. The evolution of such a void for $\lambda=0.65$ is shown in Figure 7. We also present the compact support solution for the initially parabolic (symmetric) void at 


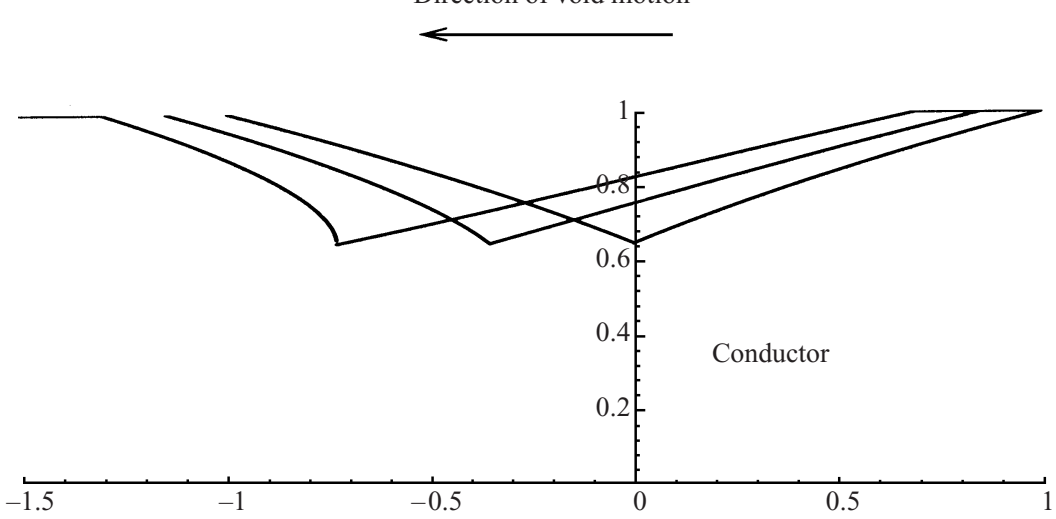

FIGURE 7. Evolution of the ZST void solution (6.26) for $\lambda=0.65$. The profile is shown at times $t=0, t=0.15$ and $t=0.309 \approx t^{*}$.

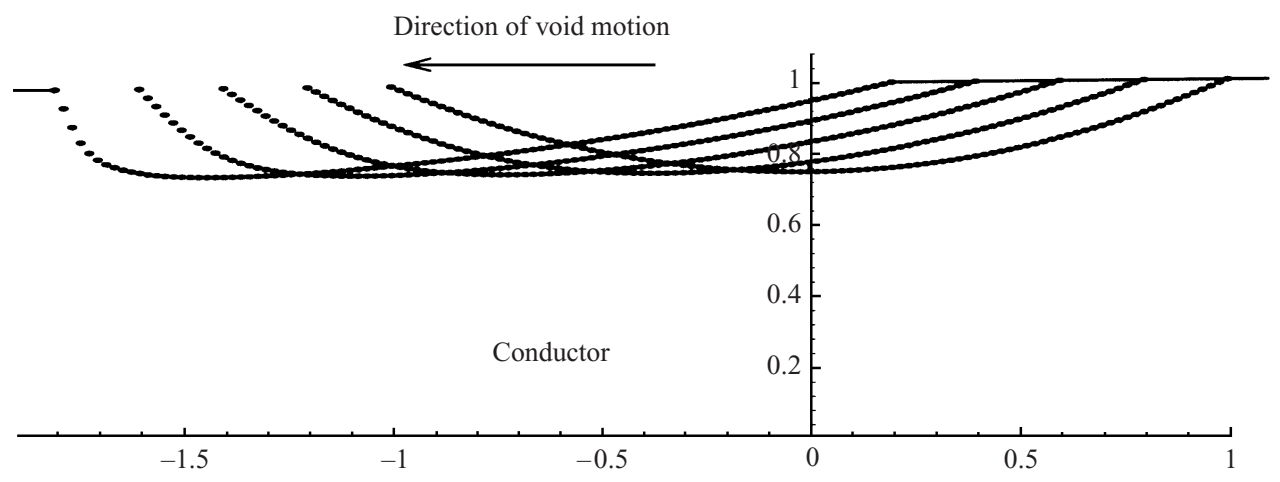

FIGURE 8. Evolution of the ZST void solution represented by (6.27) for $\lambda=3 / 4$. The profile is shown at times $t=0,0.2,0.4,0.6,0.8$.

a boundary, for comparison:

$$
f_{0}(x)= \begin{cases}1 & |x|>1 \\ \lambda+x^{2}(1-\lambda) & -1<x<1\end{cases}
$$

As mentioned, this leads to a quintic polynomial for $f$ :

$$
f=f_{0}\left(x+\frac{t}{x^{2}}\right)=\lambda+(1-\lambda)\left(x+\frac{t}{f^{2}}\right)^{2},
$$

requiring numerical solution. Figure 8 shows the evolution for $\lambda=3 / 4$ until shortly before the solution blows up with $f_{x}$ becoming infinite.

\subsubsection{Numerical time-dependent solutions}

We present solutions with $f(+\infty)=1$ and $f(-\infty)=\lambda$ for the three cases: $\lambda<1$, $\lambda>1, \lambda=1$. The numerical scheme used is semi-implicit (the $(1 / f)_{x}$ term being treated explicitly), and is based on the Crank-Nicolson scheme for the usual diffusion equation. 


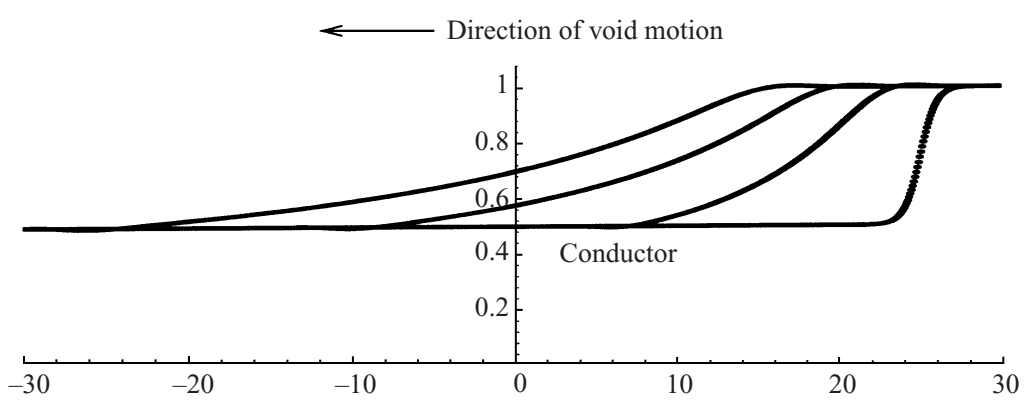

FIGURE 9. Numerical solution of (6.21) for an initial profile $f(x, 0)=\lambda+(1-\lambda)(\tanh x+1) / 2$ $(\lambda=0.5)$ and $\sigma^{*}=0.1$. The profile is shown at times $t=0,4,8,12$ (in the dimensionless units). Note the nonuniform scaling of horizontal and vertical axes, to make the void shape evolution easier to see.

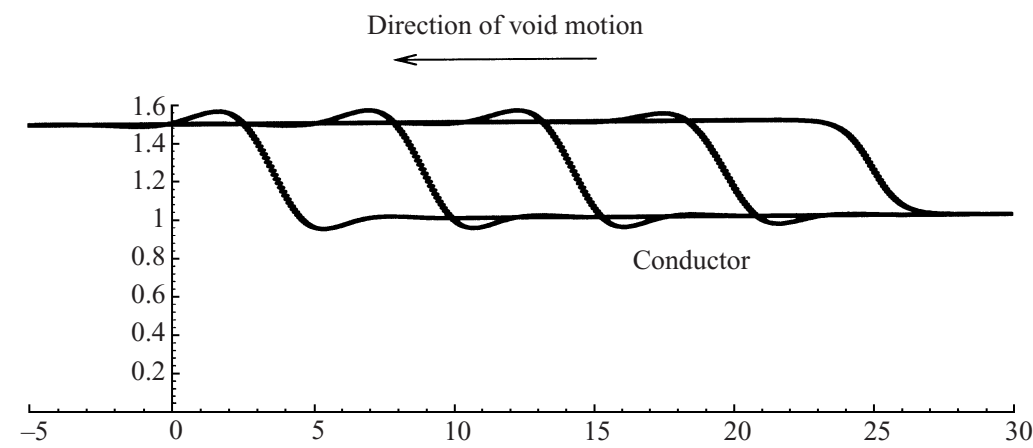

Figure 10. Numerical solution of (6.21) for an initial profile $f(x, 0)=\lambda+(1-\lambda)(\tanh x+1) / 2$ $(\lambda=1.5)$ and $\sigma^{*}=0.1$. The profile is shown at times $t=0,8,16,24,32$ (in the dimensionless units).

Figures 9 and 10 show the evolution of semi-infinite voids for which the initial shape of the conductor profile is given by

$$
f(x, 0)=\lambda+\frac{1-\lambda}{2}(\tanh x+1),
$$

so that $f(+\infty, 0)=1$ and $f(-\infty, 0)=\lambda$. For Figure $9 \lambda$ is 0.5 ; numerically this appears to be rather a stable situation, the steep initial profile smoothing out as time increases. Surface tension becomes less and less important for large times.

Figure 10 shows the analogous solution for the case $\lambda=1.5$. The initial profile develops a small peak in advance of the moving disturbance and a small pit behind it, and the advancing wavefront steepens. It appears that this solution is approaching a travellingwave form; this is discussed further in $\S 6.1 .4$ below.

Finally, Figure 11 shows a solution for which $f( \pm \infty)=1$. The initial disturbance is symmetric: $f(x)=1-0.6 \exp \left((x-25)^{2}\right)$.

We note that if we take higher values of the surface tension parameter $\sigma^{*}$, the damping (stabilising) effect in figure 11 is severe (even with the value chosen it is significant); one has to take $\sigma^{*}$ quite small to get any kind of interesting behaviour. Nonetheless, the behaviour displayed in Figures 10 and 11 is intrinsically different from that of the ZST 


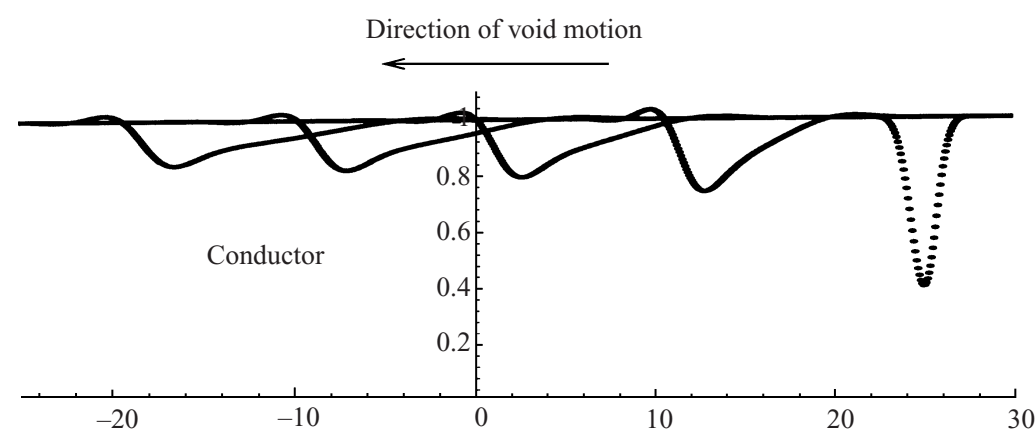

FIGURE 11. Numerical solution of (6.21) for an initial profile $f(x)=1-0.6 \exp \left((x-25)^{2}\right)$ and $\sigma^{*}=0.1$. The profile is shown at times $t=0,8,16,24,32$ (in the dimensionless units).

$\left(\sigma^{*}=0\right)$ model considered in $\S 6.1 .2$; in particular, the model with $\sigma^{*}>0$ will not permit the profile to develop an actual shock. Figure 9 does display the same characteristics as the ZST model though, and in fact the long-time asymptotics of solutions like this are given by the ZST model. This is considered in $\S 6.1 .4$ below.

\subsubsection{Long time asymptotics of solutions to the model (6.21)}

A travelling wave solution of (6.21) in the case $\lambda>1$

We again assume boundary conditions $f(\infty)=1, f(-\infty)=\lambda$ (where $\lambda$ is positive but may be less than, equal to, or greater than 1) and seek a solution of the form

$$
f(x, t)=F(x+c t)
$$

for some constant $c$. The equation for $F$ may be integrated once, giving

$$
c F+\frac{1}{F}+\sigma^{*} F^{\prime \prime \prime}=1+c,
$$

using the boundary condition at plus infinity. The condition at minus infinity then gives a relation between $\lambda$ and $c$, namely $c=1 / \lambda$ (so $c>0$, and if waves exist they travel from right to left). Multiplying through by $F^{\prime}$ and integrating again we find

$$
\sigma^{*} \int_{-\infty}^{\infty}\left(F^{\prime \prime}\right)^{2} d x=\log c+\frac{1}{2 c}\left(1-c^{2}\right) .
$$

The left-hand side is clearly positive, and the right-hand side is positive for $0<c<1$ and negative for $c>1$. Hence a necessary condition for a nontrivial travelling-wave solution is $0<c<1$, i.e. $\lambda>1$, which we might have anticipated from the simulations of Figures $9-11$.

Now consider the asymptotics as $x \rightarrow \infty$ and $F$ approaches 1 . In the neighbourhood of the point $x=X_{0}$ (where $X_{0} \gg 1$ ) we linearise about $F=1$ by introducing the small parameter $\epsilon \ll 1$ and writing $F=1+\epsilon F_{1}+\cdots$. Substituting this asymptotic expansion 
into (6.28) we find that

$$
\begin{aligned}
& F \sim 1+\epsilon\left(a_{1} \exp \left(\alpha\left(x-X_{0}\right)\right)+\exp \left(-\alpha\left(x-X_{0}\right) / 2\right)\left(a_{2} \cos \left(\sqrt{3} \alpha\left(x-X_{0}\right) / 2\right)+\right.\right. \\
& \left.\left.\qquad a_{3} \sin \left(\sqrt{3} \alpha\left(x-X_{0}\right) / 2\right)\right)\right)+\cdots, \\
& \alpha=\left(\frac{1-c}{\sigma^{*}}\right)^{1 / 3},
\end{aligned}
$$

where $a_{1}, a_{2}$ and $a_{3}$ are constants. Clearly $a_{1} \equiv 0$ in order that the asymptotic solution decays as $x \rightarrow \infty$. We can perform a similar asymptotic analysis as $x \rightarrow \infty$ and $F$ approaches $\lambda$ by considering the behaviour in the vicinity of the point $x=-X_{1}\left(X_{1} \gg 1\right)$. We find

$$
\begin{aligned}
& F \sim 1+\delta\left(b_{1} \exp \left(-\beta\left(x+X_{1}\right)\right)+\exp \left(\beta\left(x+X_{1}\right) / 2\right)\left(b_{2} \cos \left(\sqrt{3} \beta\left(x+X_{1}\right) / 2\right)+\right.\right. \\
& \left.\left.b_{3} \sin \left(\sqrt{3} \beta\left(x+X_{1}\right) / 2\right)\right)\right)+\cdots \\
& \beta=\left(\frac{c(1-c)}{\sigma^{*}}\right)^{1 / 3},
\end{aligned}
$$

where $\delta \ll 1$ and $b_{1}, b_{2}$ and $b_{3}$ are all constants. Requiring that the solution decay as $x \rightarrow-\infty$ forces $b_{1} \equiv 0$.

We can find numerical solutions to equation (6.28) together with the boundary conditions

$$
F \rightarrow 1 \quad \text { as } x \rightarrow \infty, \quad F \rightarrow \lambda \quad \text { as } x \rightarrow-\infty
$$

using a shooting method. In order to approximate (6.33) we use the far field asymptotic behaviour determined in (6.29) and (6.31). Note that this far field behaviour is insufficient in itself to determine a unique solution since only two of the constants $a_{i}$ and $b_{i}(i=1,2,3)$ are known; however this is easily seen to result from the fact that (6.28) is invariant under translations. It thus suffices to fix one of the other constants (say $b_{2}$ ) and shoot by changing $b_{3}$ until the boundary condition (6.29), with $a_{1}=0$, is satisfied. Changing the value at which we fix $b_{2}$ results in the same solution modulo a translation. The result of such a calculation is shown in Figure 12 (crosses), together with the long-time behaviour of a time-dependent simulation like that of figure 10. Here, larger values of $\sigma^{*}$ and $\lambda$ than those of figure 10 are used, for purposes of illustration; it is clear however that the main features of the evolution are identical. In particular, it is easily checked from figure 10 that the wavefront is moving with a speed close to the expected value of $0.666=1 / \lambda$.

\section{A similarity solution to equation (6.21) in the case $\lambda<1$}

Numerical solutions of equation (6.21) suggest that, for $\lambda<1$, the solution becomes progressively attenuated as time increases; it follows that the surface tension term becomes unimportant over long-time scales. We thus attempt to describe the long-time behaviour for this case by looking for a similarity solution of the ZST model (6.24). Formally, we can obtain the ZST limit from the full model by rescaling $x$ and $t$ with $\Gamma \gg 1$ such that

$$
x=\Gamma x^{\prime} \quad t=\Gamma t^{\prime} .
$$




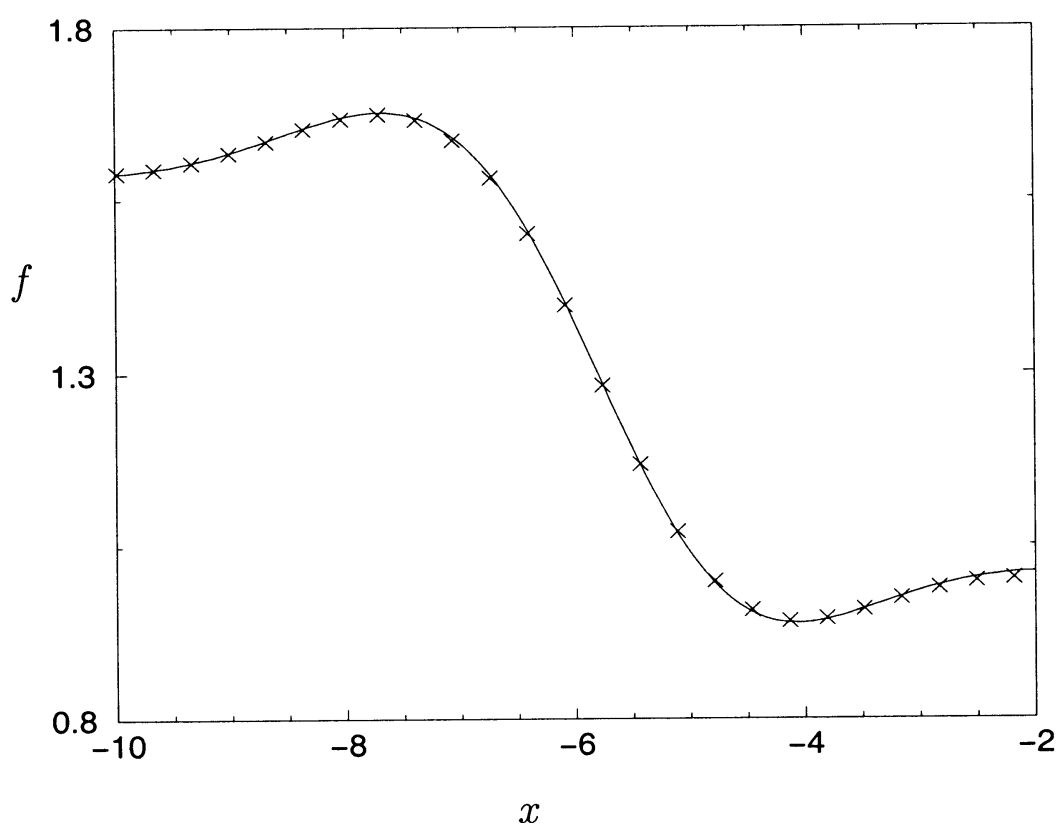

FIGURE 12. Convergence to a travelling wave solution. The travelling wave solution has been plotted with crosses while the long-time behaviour of the solution to (6.21), with a ramp profile for initial data, is shown by the solid line. Here $\sigma^{*}=0.1, \lambda=1.6$.

Performing this rescaling in (6.21), we obtain the ZST model (6.24) together with an error term of $O\left(\sigma^{*} / \Gamma^{3}\right)$. Given monotonic initial data for the full model we expect to be able to describe its long time behaviour by the rescaled model with the discontinuous initial data

$$
\left.f\right|_{t^{\prime}=0}=\lambda+(1-\lambda) H\left(x^{\prime}\right),
$$

where $H(\cdot)$ is the Heaviside step function. This has the similarity solution

$$
\left.\begin{array}{lc}
f=\lambda & \frac{x^{\prime}}{t^{\prime}}<-\frac{1}{\lambda^{2}} \\
f=\left(-\frac{t^{\prime}}{x^{\prime}}\right)^{1 / 2} & -\frac{1}{\lambda^{2}}<\frac{x^{\prime}}{t^{\prime}}<1 \\
f=1 & -1<\frac{x^{\prime}}{t^{\prime}}
\end{array}\right\} .
$$

Note that, since this solution is invariant under scalings (such as (6.34)) which preserve $x^{\prime} / t^{\prime}$, the actual size of the large parameter $\Gamma$ used in (6.34) is irrelevant. In Figure 13 we show how solutions computed to equation (6.21) converge towards the similarity solution (6.35).

\section{Surface tension and other effects}

We have neglected the effects of surface tension at several points in this paper: in the brief discussion of finger-type solutions at the start of $\S 5$; when considering small voids in a strip $(\S 5.1)$; in our compact support solutions of $\S 6.1 .2$; and in the stability analyses 


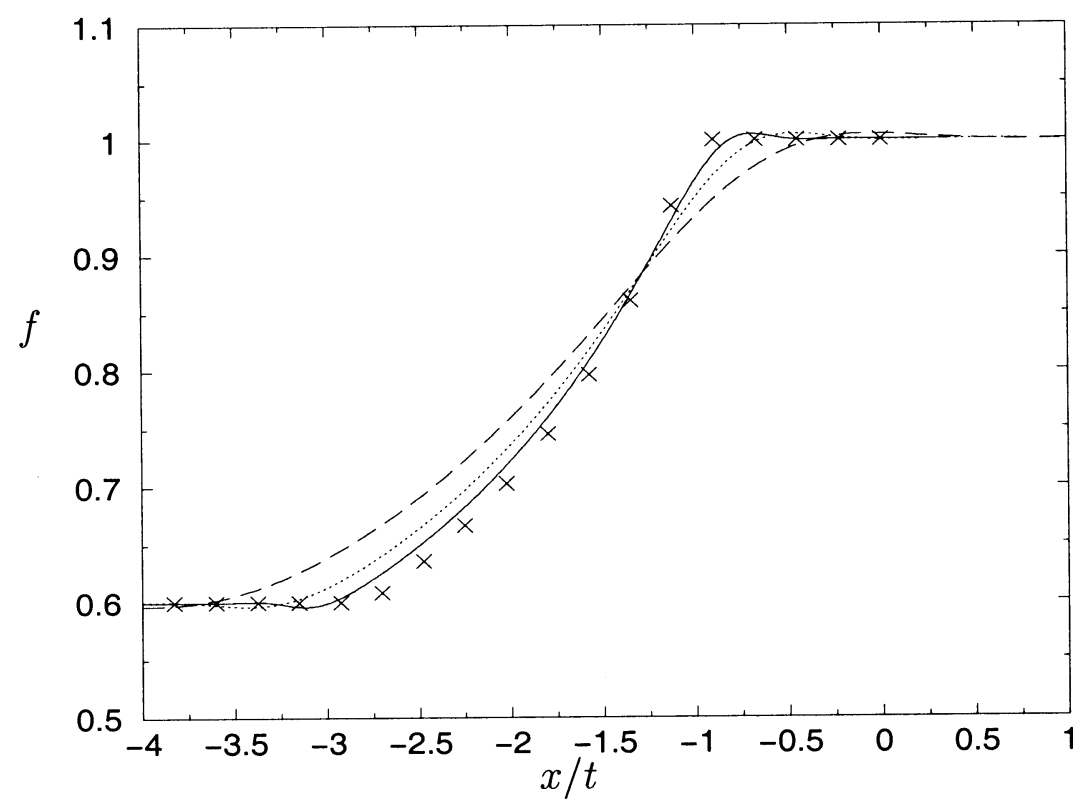

FIGURE 13. Convergence to a similarity solution. The similarity solution has been plotted with crosses while the behaviour of the solution to (6.21), starting from inital data $f(x, 0)=\lambda+(1-$ $\lambda)(\tanh x+1) / 2$, is shown at times $t=3.0, t=6.0$ and $t=12.0$ by dashed, dotted and solid lines respectively. Here $\sigma^{*}=0.1, \lambda=0.6$.

of $\S$ 4.1.3. It is therefore of interest to consider what effect surface tension has on possible solution shapes.

In the context of the finger solutions, it was observed by Ben Amar [2] that inclusion of the surface tension term in the kinematic boundary condition destroyed the travelling wave solution, numerically. We can give a heuristic argument as to why this should be so, by thinking about the change in the normal velocity into the void $v_{n}$ brought about by inclusion of the $\sigma \kappa_{s s}$ term. If $\theta$ is the angle made by the tangent to the finger boundary with the negative $x$-axis (Figure 5) then $\theta$ varies monotonically between 0 and $2 \pi$ (Figure 14 (a)), and $\kappa_{s s}=\theta_{\text {sss }}$ (Figure 14 (d)). From the sign changes of $\theta_{\text {sss }}$ we see that if one seeks to add surface tension to the finger shape of Figure 5, the effect is to give a positive contribution to $v_{n}$ over some range of $\theta,|\theta|<\theta^{*}$ (say), and a negative contribution (which rapidly tends to zero) for $|\theta|>\theta^{*}$. This suggests that surface tension wants to make the nose of the finger bulbous (a tadpole-shaped finger), and leads to the distinct possibility that a pre-existing finger-shaped void may break up, a bulbous head forming which leaves the tail behind. Thus, it is probable that surface tension effects have important implications for solutions of this kind.

No such numerical difficulties arise for the bubble-in-a-strip solutions of Ben Amar [2]; surface tension effects can be included without difficulties, and without greatly perturbing the boundary. If the ZST shape is known, an argument similar to that given above to explain the destruction of the finger solution may be used to explain the change in the void shape when surface tension is added to the problem. Since the ZST shapes calculated are very nearly circular, moderate values of $\sigma$ do not much affect the shape. 

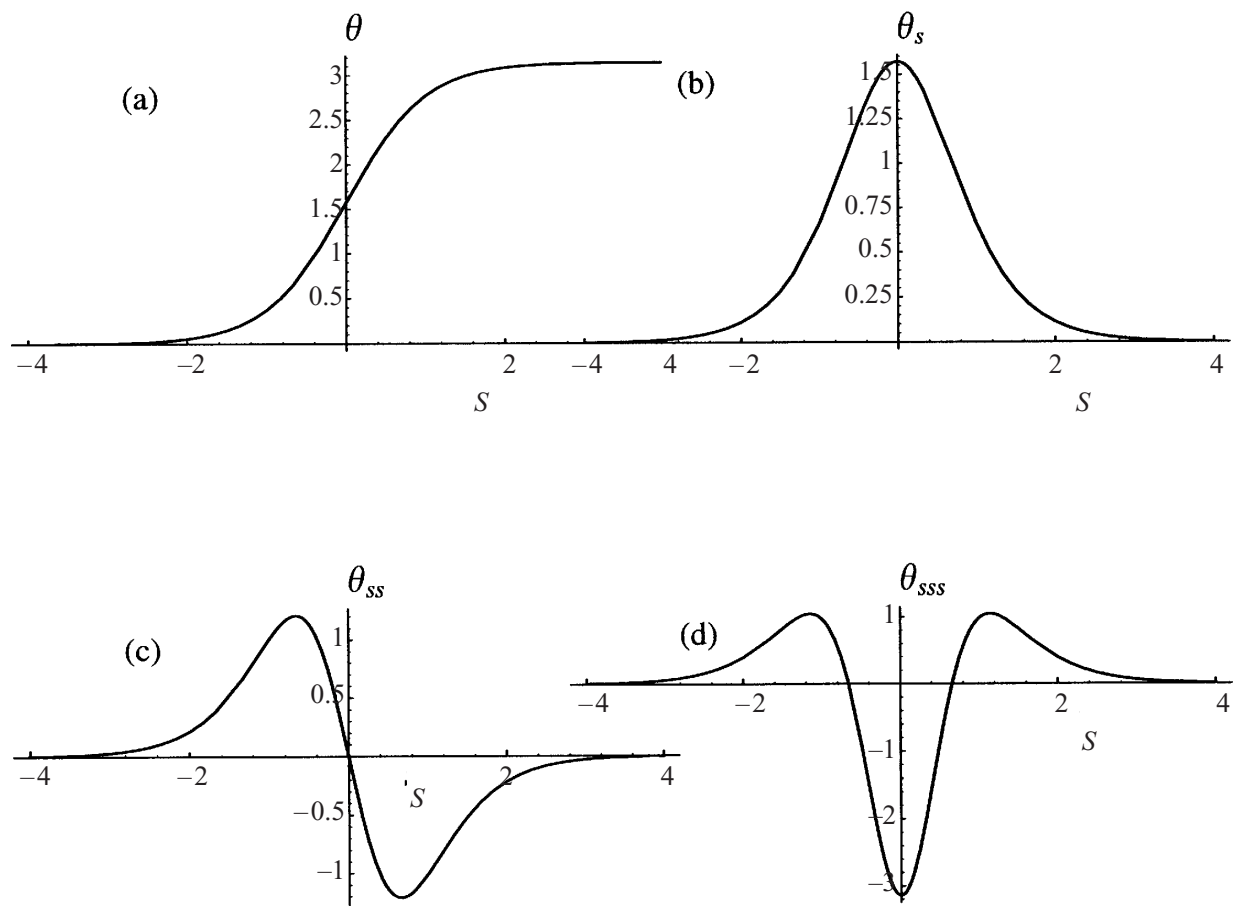

Figure 14. The angle $\theta(s)$ made by the tangent to the finger void (a), and its first three derivatives with respect to arclength $s((\mathrm{~b}),(\mathrm{c}),(\mathrm{d})$, respectively).

For the compact support solutions given in $\S 6.1 .2$ we obviously expect surface tension to have some regularising effect at the edges of the support $(x= \pm 1-t)$, since $\kappa$ is discontinuous there. Similarly, it must be important near the 'point' on the solution of Figure 7. Despite observing qualitatively the same asymmetric 'steepening' behaviour as with the more complicated model (6.21), a comparison of Figures 11 and 8 suggests that real voids having nearly-compact support will spread their initial support under the influence of the diffusive surface tension term, though this effect may be less pronounced than in our numerical solutions. In reality, then, we expect a competition between this stabilising diffusion, and the steepening effect due to the electric field. If the surface tension effect is sufficiently small relative to the electric field effect, the advancing side of the void will steepen sufficiently that the model (6.21) becomes invalid (this will happen if, for example, $f_{x}$ becomes $O(1 / \delta)$; that circuit failure occurs implies that steepening due to the electric field frequently does 'win'). In this event, a new model is needed to describe the subsequent evolution. Recent numerical simulations of the full model [11,27] suggest that with isotropic surface tension, the void subsequently takes the shape of a finger, of roughly uniform thickness, growing parallel to the electric field direction. To obtain the catastrophic circuit-breaking fingering observed in the experiments of Arzt et al. [1], it is conjectured [11] that some degree of surface anisotropy is needed in the model (see below). We note however that our 'long wavelength' isotropic model captures the asymmetric steepening seen prior to circuit failure in experiments; Arzt et al. [1] state: "Our main observation, which we think is significant for the failure mechanism, pertains to the fact that fatal voids 
have a typical slit or wedge shape with a pronounced asymmetry". In fact, our simplest ZST compact support solution bears considerable qualitative resemblance to some of the experimental voids of Arzt et al. [1], prior to breakdown, suggesting that sometimes (at high currents) surface tension effects are not so important in determining the early void behaviour.

If one considers the boundary condition $v_{n}=\sigma \kappa_{s s}$, which can be thought of as the large$\sigma$ limit of the problem, one has a problem in which the free boundary is evolving under the action of surface diffusion alone (the problem for the electric potential $\phi$ decouples and must be solved on the moving domain subject to the condition $\partial \phi / \partial n=0$ on the boundary). This motion by surface diffusion is a classical mathematical problem which has been extensively studied (for example, see Elliott \& Garcke [7] and the references therein). It is area-preserving and perimeter-decreasing. It has been proved that if the initial boundary curve is close to a circle (in some precise sense) then one has global existence of solutions, and that in this case solutions evolve to circles (the only stable equilibria) [7], which is analogous to our result of $\S 4.1 .4$, that circles are the only travelling-wave solutions to the infinite domain problem. For arbitrary initial boundary shapes [7] also proves local-in-time existence of solutions. However they conjecture that for certain initial shapes, self-intersection of the boundary curve can occur, which for the electromigration problem corresponds to a void breaking up. This is possibly slightly counter-intuitive, since one tends to think of surface tension always having a regularising effect, whereas void break-up seems rather an irregular occurrence. The argument they give is along the same lines as that which we advance to explain the non-existence of a travelling wave finger (and indeed, the possibility of the finger breaking up) when $\sigma>0$, that is, looking at the sign of $v_{n}$ produced by a hypothesised initial boundary curve. We expect that having $\sigma>0$ in the model can lead to changes in void topology in more general situations; this has been observed in numerical simulations $[15,25]$, and it is possibly one of its most important effects. Note however that topology change may also be possible for the ZST problem, as tentatively discussed in the stability analysis of $\S 4.1 .3$.

Finally, we mention the work of Gungor et al. [11, 12], which incorporates the additional effects of surface anisotropy and mechanical stress. In Gungor \& Maroudas [11] the surface tension is taken to be isotropic, but the mobility $M$, which premultiplies both terms in the kinematic boundary condition (2.3), is anisotropic. A phenomenological relation of the form $M=M_{\min }\left(1+A \cos ^{2}(m(\theta+\phi))\right)$ is assumed, where $\theta$ is the angle formed by the local tangent to the surface and the direction of the applied electric field, $A$ measures the strength of the anisotropy, $m=1,2$ or 3 is the number of crystallographic directions which correspond to 'fast paths' for surface diffusion, and $\phi$ is the orientation of one of these directions with respect to the electric field direction. Numerical simulations of this model show voids which exhibit faceting, and which, for suitable choices of the parameters $A$ and $\phi$, can traverse the whole width of the conductor, leading to circuit failure. In Gungor et al. [12] the effect of mechanical stress on the void evolution is considered. The authors argue that in a passivated metal film (where the exposed surfaces of the conductor are covered with a layer of glass to suppress electromigration damage), significant mechanical stress can build up within the conductor, leading to crack formation as a possible failure mechanism. They formulate a model which addresses this issue through the inclusion of linear elasticity, in addition to the anisotropic surface effects mentioned above. Numerical 
simulations indicate that if high stresses do accumulate, the void evolution can change qualitatively, with crack-like features emerging from highly-curved points on the void boundary.

\section{Conclusions}

There are two main aims of this paper. First, we wish to give a more mathematical treatment of a problem which has already been much-studied from a physical perspective. To this end, we develop a complex variable approach in $\S 3$ which is applied in $\S 4$ and $\S 5.1$, and also use methods of formal asymptotics in $\S 5.1$ and $\S 6$. Because we wish to appeal to a mathematics as well as a physics readership, some of the paper is review; however, we hope we are clear about which results are not new. In addition, our non-new results are presented in our complex variable framework, which has not been considered in any detail before, or (as with our linear stability analyses of $\S 4.1 .3$ ) are analytical rather than numerical.

Secondly, we wish to present our truly new work on the problem. We give several novel results: it is proved that the circular travelling-wave is the only solution of finite void area in the unbounded geometry (with or without surface tension effects); an asymptotic treatment of small voids in a conducting strip is carried out; and a comprehensive asymptotic treatment of voids at the boundary of a conducting strip is presented, along with several examples of solutions. We also consider the possibility of static void solutions, observing that in this case the problem is identical to the classical Hele-Shaw problem. Our asymptotic model (6.21) in particular represents a considerable simplification of the full problem, yet retains most of the salient features, in particular, nonlinear effects. Its drawback is, of course, its inability to describe the conductor failure, since as the degree of void asymmetry increases, $f_{x}$ becomes very large and the model breaks down. A different model is required to describe the subsequent evolution, the development of which is one of our future goals.

\section{Appendix A Units of the various physical quantities}

This appendix gives the SI units of the physical quantities and parameters referred to in this paper, for ease of reference.

$\begin{array}{llll}\text { Electric potential } \phi & : \mathrm{kg} \mathrm{m}^{2} \mathrm{~s}^{-2} \mathrm{C}^{-1} & \text { Void curvature } \kappa & : \mathrm{m}^{-1} \\ \text { Effective charge } Z^{*} e & : \mathrm{C} & \text { Adatom mobility } M & : \mathrm{m} \mathrm{s} \mathrm{kg}^{-1} \\ \text { Atomic volume } \omega & : \mathrm{m}^{3} & \text { Surface tension } \gamma & : \mathrm{kg} \mathrm{s}^{-2} \\ \text { Field at infinity } E_{\infty} & : \mathrm{kg} \mathrm{m} \mathrm{s}^{-2} \mathrm{C}^{-1} & A=Z^{*} e M & : \mathrm{C} \mathrm{m} \mathrm{s} \mathrm{k}^{-1} \\ B=M \omega \gamma & : \mathrm{m}^{4} \mathrm{~s}^{-1} & & \end{array}$

\section{Acknowledgements}

L.J.C. and G.W.R. gratefully acknowledge financial support from the European Community, in the form of postdoctoral fellowships. 


\section{References}

[1] Arzt, E., Kraft, O., Nix, W. D. \& Sanchez, J. E. (1994) Electromigration failure by shape change of voids in bamboo lines. J. Appl. Phys. 76(3), 1563-1571.

[2] Ben Amar, M. (1999) Void electromigration as a moving boundary problem. Physica D 134(2), 275-286.

[3] Bradley, R. M., Mahadevan, M. \& Wu, K. (1999) Exact results on void growth in a model of electromigration. Phil. Mag. B, 79(2), 257-268.

[4] Carrier, G. F., Krook, M. \& Pearson, C. E. (1966) Functions of a Complex Variable. McGrawHill.

[5] Davi, F. \& Gurtin, M. (1990) On the motion of a phase interface by surface diffusion. J. Appl. Math. and Phys. (Z.A.M.P.) 41, 782-811.

[6] Davis, P. J. (1974) The Schwarz function and its applications. Carus Math. Monographs 17.

[7] Elliott, C. M. \& Garcke, H. (1997) Existence results for diffusive surface motion laws. Adv. Math. Sci. and Appl. 7(1), 467-490.

[8] Entov, V. M., Etingof, P. I. \& KLeinbock, D. Ya. (1993) Hele-Shaw flows with a free boundary produced by multipoles. Euro. J. Appl. Math. 4, 97-120.

[9] Galin, L. A. (1945) Unsteady filtration with a free surface Dokl. Akad. Nauk. S.S.S.R. 47, 246-249 (in Russian).

[10] Gradshteyn, I. S. \& Ryzhik, I. M. (1980) Table of Integrals, Series and Products (corrected and enlarged edition). Academic Press.

[11] Gungor, M. R. \& Maroudas, D. (1999) Theoretical analysis of electromigration-induced failure of metallic thin films due to transgranular void propagation. J. Appl. Phys. 85(4), 2233-2246.

[12] Gungor, M. R., Maroudas, D. \& Gray, L. J. (1998) Effects of mechanical stress on electromigration-driven transgranular void dynamics in passivated metallic thin films. Appl. Phys. Lett. 73(26), 3848-3850.

[13] Ho, P. S. (1970) Motion of inclusion induced by a direct current and a temperature gradient. J. Appl. Phys. 41, 64.

[14] Howison, S. D. (1992) Complex variable methods in Hele-Shaw moving boundary problems. Euro. J. Appl. Math. 3, 209-224.

[15] Li, Z. L., ZHAO, H. K. GAO, H. J. (1999) A numerical study of electro-migration voiding by evolving level set functions on a fixed cartesian grid. J. Computational Phys. 152(1), 281-304.

[16] Mclean, J. W. \& Saffman, P. G. (1981) The effect of surface tension on the shape of fingers in a Hele-Shaw cell. J. Fluid Mech. 102, 455-469.

[17] Mahadevan, M. \& Bradley, R. M. (1996) Stability of a circular void in a passivated, currentcarrying metal film. J. Appl. Phys. 79(9), 6840-6847.

[18] Mahadevan, M. \& Bradley, R. M. (1999) Simulations and theory of electromigration-induced slit formation in unpassivated single crystal metal lines. Phys. Rev. B 59(16), 11037-11046.

[19] Mahadevan, M., Bradley, R. M. \& Debierre, J.-M. (1999) Simulations of an electromigrationinduced edge instability in single crystal metal lines. Europhys. Lett. 45(6), 680-685.

[20] Millar, R. F. (1990) Inverse problems for a class of Schwarz functions. Complex Variables, 15, 1-10.

[21] Mullins, W. W. (1957) Theory of thermal grooving. J. Appl. Phys. 28, 333-339.

[22] Ockendon, H. \& Tayler, A. B. (1983) Inviscid Fluid Flows. Applied Mathematical Sciences, 43. Springer-Verlag.

[23] Saffman, P. G. \& TAYloR, G. I. (1958) The penetration of a fluid into a porous medium or Hele-Shaw cell containing a more viscous liquid. Proc. Roy. Soc. Lond. A. 245, 312-329.

[24] Schimschak, M. \& Krug, J. (1997) Surface electromigration as a moving boundary value problem. Phys. Rev. Lett. 78(2), 278-281.

[25] SchimschaK, M., Krug, J. Electromigration-induced breakup of two-dimensional voids. Phys. Rev. Lett. 80(8), 1674-1677.

[26] Shapiro, H. S. (1984) Domains allowing exact quadrature identities for harmonic functions - an 
approach based on PDE. In: P. L. Butzer et al. (eds.), Anniversary Volume on Approximation Theory and Functional Analysis International Series of Numerical Mathematics, 65, pp. 335354. Birkhäuser-Verlag.

[27] Shelley, M. J. (1999) Unpublished.

[28] Sornette, D. \& Vanneste, C. (1992) Dynamics and memory effects in rupture of thermal fuse networks. Phys. Rev. Lett. 68(5), 612-615.

[29] Sornette, D. \& Vanneste, C. (1994) Dendrites and fronts in a model of dynamical rupture with damage. Phys. Rev. E. 50(6), 4327-4345.

[30] SuO, Z., WANG, W. \& YANG, M. (1994) Electromigration instability: Transgranular slits in interconnects. Appl. Phys. Lett. 64(15), 1944-1946.

[31] van Dyke, M. (1974) Perturbation Methods in Fluid Dynamics. Parabolic Press.

[32] Wang, W., SuO, Z. \& HaO, T.-H. (1996) A simulation of electromigration-induced transgranular slits. J. Appl. Phys. 79(5), 2394-2403. 